\title{
Predicted UV properties of very metal-poor starburst galaxies ${ }^{\star}$
}

\author{
A. Raiter ${ }^{1}$, D. Schaerer ${ }^{2,3}$, and R. A. E. Fosbury ${ }^{4}$ \\ ${ }^{1}$ European Southern Observatory, Karl-Schwarzschild-Strasse 2, 85748 Garching bei München, Germany \\ e-mail: araiter@eso.org \\ 2 Geneva Observatory, University of Geneva, 51, chemin des Maillettes, 1290 Versoix, Switzerland \\ e-mail: daniel.schaerer@unige.ch \\ 3 Observatoire Midi-Pyrénées, Laboratoire d'Astrophysique, UMR 5572, 14 Avenue E. Belin, 31400 Toulouse, France \\ 4 ST-ECF, Karl-Schwarzschild Str. 2, 85748 Garching bei München, Germany \\ e-mail: rfosbury@eso.org
}

Received 18 June 2010 / Accepted 11 August 2010

\section{ABSTRACT}

\begin{abstract}
Aims. We study the expected properties of starburst galaxies in order to provide the point of reference for interpretation of high- $z$ galaxy surveys and of very metal-poor galaxies. We concentrate mainly on the UV characteristics such as the ionizing spectra, the UV continuum, the Ly $\alpha$ and He II $\lambda 1640$ line and two-photon continuum emission.

Methods. We use evolutionary synthesis models covering metallicities from Pop III to solar and a wide range of IMFs. We also combine the synthetic SEDs with the CLOUDY photoionization code for more accurate predictions of nebular emission, and to study possible departures from case B assumed in the synthesis models.

Results. The ionizing fluxes, UV continuum properties, and predicted Ly $\alpha$ and He II $\lambda 1640$ line strengths are presented for synthesis models covering a wider range of parameter space than our earlier calculations. Strong departures from case B predictions are obtained for $\operatorname{Ly} \alpha$ and $2 \gamma$ continuum at low metallicities. At low nebular densities both are shown to be enhanced proportionally to the mean energy carried by the Lyman continuum photons emitted by the ionizing source. Larger Ly $\alpha$ equivalent widths are therefore predicted at low metallicity. The He II $\lambda 1640$ line can be weaker than case B predicts (in terms of flux as well as the equivalent width) due to its ionization parameter dependence and to the enhanced underlying $2 \gamma$ continuum.

Conclusions. Our results have implications for the interpretation of star-forming metal-poor and/or high redshift galaxies, for galaxies among the Ly $\alpha$ emitters (LAE) and Lyman Break galaxy (LBG) populations, and for searches of Population III stars in the distant Universe.
\end{abstract}

Key words. galaxies: high-redshift - galaxies: evolution - galaxies: starburst - early Universe

\section{Introduction}

Over the last decade, the execution of deep multi-band imaging surveys like GOODS (Vanzella et al. 2005, 2006, 2008, 2009; Popesso et al. 2009; Balestra et al. 2010) has resulted in catalogues of significant numbers of galaxies with photometric redshifts greater than five when the Universe was only a little over a Gyr old. In some cases, these redshifts have been spectroscopically confirmed by the detection of $\mathrm{Ly} \alpha$ in emission and/or the presence of an identifiable Lyman break. The availability of an increasing sensitivity in the NIR, notably with the newlyinstalled WFC3 camera in the HST and with the JWST in the near future, opens the possibility of selecting high quality candidates up to and beyond a redshift of 10 . This is the epoch where it can be expected that stellar populations have a very low metallicity, which may result in an excess of hot, high mass stars radiating strongly in the Lyman continuum.

To provide appropriate spectral templates for such metalpoor star-forming galaxies and to predict the observable properties of starbursts with primordial and more evolved chemical compositions Schaerer $(2002,2003)$ has computed new evolutionary synthesis models and has demonstrated the importance of nebular emission (lines and continua) at low metallicity. These

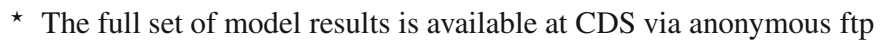
to cdsarc.u-strasbg.fr $(130.79 .128 .5)$ or via

http://cdsarc.u-strasbg.fr/viz-bin/qcat?J/A+A/523/A64 and other studies (see e.g. Tumlinson \& Shull 2000; Tumlinson et al. 2001; Bromm et al. 2001) have in particular highlighted the use of strong Ly $\alpha$ emission and nebular He II emission to search for objects containing Population III (hereafter Pop III) stars. Since then, various searches for the He II $\lambda 1640$ signature from Pop III have been undertaken at different redshifts, yielding so far non-detections and interesting upper limits on the Pop III star-formation rate density (see Schaerer 2008; Nagao et al. 2008, and references therein). Furthermore, among the numerous surveys for Ly $\alpha$ emitters at different redshifts, some studies have found objects with apparently unusually strong Ly $\alpha$ emission (high equivalent widths), which could be indicative of very metal-poor (even Pop III) stellar populations or unusual IMFs (see e.g. Malhotra \& Rhoads 2002; Yamada et al. 2005). Other groups have invoked "unusual" IMFs, extremely metalpoor stellar populations, and/or leakage of Lyman continuum radiation to explain the apparently very blue UV slopes found for some very high redshift $(z \sim 7)$ galaxies (Bouwens et al. 2010). However, the significance of these results is questionable, and the present data does not require such "non-standard" assumptions (Schaerer \& de Barros 2010; Finkelstein et al. 2010). In any case, it is of interest to examine how reliable some of the major observables predicted by standard evolutionary synthesis are.

Indeed, a shortcoming of evolutionary synthesis models such as the ones mentioned above is that they calculate nebular emission in an approximate manner assuming simplified physics, 
Table 1. Summary of IMF model parameters.

\begin{tabular}{|c|c|c|c|c|c|c|c|c|}
\hline Model ID & Colour code & $M_{\text {low }}$ & $M_{\text {up }}$ & $\alpha$ & $M_{\mathrm{c}}$ & $\sigma$ & Reference/comment & ID in files \\
\hline Salpeter & black & 1 & 100 & 2.35 & & & A in Schaerer (2003) & $\mathrm{S}$ \\
\hline B & green, dashed & 1 & $500^{a}$ & 2.35 & & & B in Schaerer (2003) & B \\
\hline $\mathrm{C}$ & cyan, dashed & 50 & $500^{a}$ & 2.35 & & & $\mathrm{C}$ in Schaerer (2003) & $\mathrm{C}$ \\
\hline Scalo & blue & 1 & $500^{a}$ & $2.7^{b}$ & & & Scalo (1986) & $\mathrm{Sc}$ \\
\hline TA & red & 1 & $500^{a}$ & & 10. & 1.0 & A in Tumlinson (2006) & TA \\
\hline TB & magenta & 1 & $500^{a}$ & & 15. & 0.3 & B in Tumlinson (2006) & TB \\
\hline TE & yellow & 1 & $500^{a}$ & & 60. & 1.0 & E in Tumlinson (2006) & TR \\
\hline L05 & blue, dashed & 1 & 100 & & 5. & & Larson (1998) & 10 \\
\hline
\end{tabular}

Notes. Model ID is the label used in the figures, colour code the colour and linestyle. Note: the definition of $M_{\mathrm{c}}$ and $\sigma$ is as in Tumlinson (2006). In particular sigma is the variance in $\ln (m)$, not $\log (m) !^{(a)}$ For metallicities $Z \geq 0.0004=1 / 50 Z_{\odot}$, we adopt $M_{\text {up }}=120 M_{\odot}$, the maximum mass for which Geneva stellar evolution tracks are available. ${ }^{(b)}$ Power-law exponent for $M \geq 2 M_{\odot}$.

such as case B recombination theory (cf. Osterbrock \& Ferland 2006), and constant emissivities for adopted constant values of the electron temperature and density in the $\mathrm{H}$ II region surrounding the starburst. In fact, as demonstrated in this paper, significant departures from case B are expected at low metallicities leading to stronger Ly $\alpha$ emission, and the strength of nebular He II emission predicted by full photoionization models can be reduced with respect to simple recombination theory. Indeed these physical effects, related to an increased importance of collisional effects at low metallicity due to lower radiative cooling and harder ionizing spectra - for Ly $\alpha$ - and due to competition between $\mathrm{H}$ and $\mathrm{He}$ for ionizing photons - for the intensity of $\mathrm{He} \mathrm{II/H} \mathrm{-} \mathrm{have} \mathrm{been} \mathrm{known} \mathrm{for} \mathrm{a} \mathrm{while} \mathrm{in} \mathrm{studies} \mathrm{of}$ metal-poor H II regions (cf. Davidson \& Kinman 1985; Stasińska \& Schaerer 1999; Luridiana et al. 2003) and planetary nebulae (Stasińska \& Tylenda 1986). Panagia (2002, 2005) has recently explored photoionization models for primordial nebulae. However, the importance of the above effects for the UV emission lines has so far not been thoroughly examined, in particular in the context of metal-poor and distant starburst galaxies and using up-to-date evolutionary synthesis models. The photoionization models presented here, combined with our evolutionary synthesis models, are intended to provide a framework within which to improve our knowledge of primeval galaxies and related objects.

Another limitation of the synthesis models of Schaerer (2002, 2003) concerns the initial mass function (IMF). For simplicity, three different choices of the IMF were adopted for the bulk of the calculations in these papers. However, different IMFs have been suggested in other studies related to Pop III and early stellar generations, and considerable uncertainties remain on the true shape of the IMF in the early Universe and its dependence (or not) on physical parameters. To enable the examination of the effects of a broader choice of IMFs on the expected observable properties of starbursts, we here extend the calculations of Schaerer (2003) to eight different IMFs. The resulting model grids, available in electronic format, should provide state-of-theart predictions for the interpretation of high redshift galaxies, to estimate their contribution to cosmic reionization, and for other topics.

The paper is structured as follows. In Sect. 2 we describe the input physics and the model calculations with our evolutionary synthesis code and with the photoionization code CLOUDY. The predictions from the synthesis models concerning the UV continuum, the ionizing flux, Ly $\alpha$ and He II $\lambda 1640$ emission are presented in Sect. 3. In Sect. 4 we discuss the results from the photoionization models using black body spectra, explain the deviations from case B and provide simple formulae to describe these effects on Ly $\alpha$. In Sect. 5 we show how to connect realistic SEDs with results from photoionization models using black body ionizing spectra. Our results and several implications are discussed in Sect. 6. The main results are summarised in Sect. 7.

\section{Modeling techniques}

\subsection{Synthesis models}

We have used the evolutionary synthesis code of Schaerer \& Vacca (1998) with the physical ingredients (stellar tracks, atmospheres, and prescriptions for nebular line and continuum emission) from Schaerer (2003). In particular these models allow us to predict the integrated properties of stellar populations at all metallicities from zero (Population III) to "normal", solarlike metallicity. The computations have been done for the same metallicities as in Schaerer (2003).

\subsubsection{Stellar initial mass function}

The main extension presented here with respect to the calculations of Schaerer (2003) concerns different assumptions regarding the stellar IMF. A wide range of IMFs has been considered, including power-law IMFs, such as the Salpeter or Scalo (1986) IMFs, log-normal IMFs, and the Larson (1998) IMF. The corresponding parameters are summarised in Table 1 . The stellar mass range is defined by the lower and upper mass cut-offs, $M_{\text {low }}$ and $M_{\text {up }}$ respectively, $\alpha$ being the slope of the power-law. The log-normal IMFs are described by the characteristic mass $M_{\mathrm{c}}$ and its dispersion $\sigma$. The cases computed here correspond to the cases A, B, and E in the chemical evolution study of Tumlinson (2006). The Larson (1998) IMF is described by a single parameter, its characteristic mass $M_{\mathrm{c}}$. We have computed one such case, assuming the same value of $M_{\mathrm{c}}$ as Ciardi et al. (2001) in their reionization calculations. Note, that at $Z \geq 0.0004=1 / 50 Z_{\odot}$ the upper mass cut-off is set to $M_{\text {up }}=100$ or $120 M_{\odot}$ for all IMFs, since tracks for more massive stars are not available. The quantities discussed here are insensitive to assumptions on the IMF at low masses. Our absolute quantities may therefore simply be rescaled to other IMFs including e.g. an extension below $1 M_{\odot}$.

Current knowledge suggests that the IMF for massive stars is close to Salpeter with an upper limit of $M_{\text {up }} \sim 100-120 M_{\odot}$ from solar metallicity down at least to $\sim 1 / 50 Z_{\odot}$, and that a qualitative shift of the IMF towards higher characteristic masses occurs below a critical metallicity of the order of $Z_{\text {crit }} \sim 10^{-5 \pm 1} Z_{\odot}$ (Schneider et al. 2002, 2003).

\subsubsection{Star formation histories}

For each metallicity $Z$ and IMF we have computed evolutionary synthesis models for the two limiting cases of 1) an instantaneous burst; and 2) constant star formation (CSFR). Results for other star formation histories can be derived from 
the electronic files for the simple stellar population (burst) models (see Sect. 2.1.4). In both cases the calculations have been carried out with a small time step $(0.1 \mathrm{Myr})$ to ensure the accuracy of the time integrated quantities for the CSFR case. The calculations have to be carried out up to ages of $1 \mathrm{Gyr}$. This covers the allowed ages and of galaxies at redshifts $z \gtrsim 5.8$, of interest here, as well as sufficiently long star formation timescalesto reach equilibrium in various observable properties (cf. below).

\subsubsection{Nebular emission}

To include nebular emission (recombination lines and continuum processes) in our synthesis models we initially make the following "standard" simplifying assumptions (see Schaerer 2002, 2003): ionization bounded nebula, constant electron temperature and density $\left(T_{\mathrm{e}}, n_{\mathrm{e}}\right)$, and case B. Case B in particular assumes that the recombinations to the ground-state immediately yield locally another ionization, and that photoionizations occur only from the ground-state. As we will see below, the latter may not be true in very metal-poor nebulae, leading to significant changes in the predicted spectrum of hydrogen. With these assumptions both the $\mathrm{H}$ and $\mathrm{He}$ recombination lines as well as nebular continuum emission (including free-free and free-bound emission from $\mathrm{H}$, neutral $\mathrm{He}$, and singly ionized $\mathrm{He}$, and twophoton emission of $\mathrm{H}$ ) are fully specified and their luminosity is proportional to the ionizing photon flux $Q$ in the appropriate energy range. To reflect to first order the changes of the conditions in the H II regions with metallicity, the value of the line luminosity coefficient and nebular continuous emission coefficients at metallicities $Z / Z_{\odot}<10^{-3}$ are changed as in Schaerer (2003). More precisely we adopt $T_{\mathrm{e}}=30(20) \mathrm{kK}$ for lines (continua) $Z / Z_{\odot}<10^{-3}$ and $T_{\mathrm{e}}=10 \mathrm{kK}$ for higher metallicities, and a low ISM density $n_{\mathrm{e}}=100 \mathrm{~cm}^{-3}$.

For the Ly $\alpha$ luminosity we have, with the assumptions just spelled out:

$$
L_{\mathrm{B}}(\mathrm{Ly} \alpha)=\left(1-f_{\mathrm{esc}}\right) Q(\mathrm{H}) \times h v_{\mathrm{Ly} \alpha} \times \frac{\alpha_{2^{2} \mathrm{P}}^{\mathrm{eff}}}{\alpha_{\mathrm{B}}}
$$

where the index " $\mathrm{B}$ " stands for case $\mathrm{B}, \alpha_{\mathrm{B}}=\alpha_{2^{2} \mathrm{P}}^{\mathrm{eff}}+\alpha_{2^{2} \mathrm{~S}}^{\mathrm{eff}}$ is the total case $\mathrm{B}$ recombination coefficient, and $f_{\text {esc }}$ is the escape fraction of ionizing photons out of the $\mathrm{H}$ II region (or galaxy). In all Figs. shown in this paper we assume $f_{\text {esc }}=0$. In typical conditions $\alpha_{2^{2} \mathrm{P}}^{\mathrm{eff}} / \alpha_{\mathrm{B}} \approx 0.6-0.7$. In other words approximately $2 / 3$ of the Lyman continuum photons give rise to the emission of a Ly $\alpha$ photon, the assumption commonly made. Similar relations also hold for other recombination lines, such as He II $\lambda 1640$, which is of special interest here. In our "standard" synthesis models we simply assume

$$
L_{\mathrm{B}}(\mathrm{Ly} \alpha)=Q(\mathrm{H}) \times c_{1},
$$

$L_{\mathrm{B}}(\mathrm{He}$ II $\lambda 1640)=Q\left(\mathrm{He}^{+}\right) \times c_{2}$,

with $c_{1}=1.04 \times 10^{-11} \mathrm{erg}$, and $c_{2}=5.67 \times 10^{-12}(6.04 \times$ $\left.10^{-12}\right)$ erg for $Z \leq 1 / 50 Z_{\odot}\left(>1 / 50 Z_{\odot}\right)$, and for $f_{\text {esc }}=0$. The atomic data is from Hummer \& Storey (1995) for low densities (cf. Schaerer 2003), and $Q(\mathrm{H})$ and $Q\left(\mathrm{He}^{+}\right)$are the ionizing photon flux (in photon $\mathrm{s}^{-1}$ ) above 13.6 and $54 \mathrm{eV}$ respectively.

Continuous nebular emission including free-free and boundfree emission by $\mathrm{H}$, neutral $\mathrm{He}, \mathrm{He}^{+}$, and $\mathrm{He}^{+2}$, as well as the two-photon continuum of hydrogen is included as described in Schaerer (2002), assuming $T_{\mathrm{e}}=20 \mathrm{kK}$ for $Z / Z_{\odot} \leq 5 . \times 10^{-4}$ and $T_{\mathrm{e}}=10 \mathrm{kK}$ otherwise.

As we will show below (Sect. 4), a proper treatment of all relevant processes leads to significant deviations from case $B$ at very low metallicities, increasing in particular the Ly $\alpha$ luminosity, $L(\operatorname{Ly} \alpha)$. In this case the $L(\operatorname{Ly} \alpha)$ can be rewritten as

$L(\operatorname{Ly} \alpha)=L_{\mathrm{B}}(\operatorname{Ly} \alpha) \times P \times \frac{\tilde{f}_{\text {coll }}}{2 / 3}$,

where $P$ and $\tilde{f}_{\text {coll }}$ are terms describing the mean photon energy in the Lyman continuum, and accounting for for collisional effects at high density. To compute the $\operatorname{Ly} \alpha$ equivalent width $W(\operatorname{Ly} \alpha)$ we proceed as in Schaerer $(2002,2003)$, where we use the continuum flux at $1215.67 \AA$ obtained from linear interpolation of the total (stellar + nebular) continuum (in log) between 1190 and $1240 \AA$, chosen to avoid underlying stellar Ly $\alpha$ absorption and other absorption lines if present. The predicted stellar Ly $\alpha$ absorption is small compared to the emission, except for "post-starburst" phases (see e.g. Fig. 3 in Schaerer \& Verhamme 2008).

\subsubsection{Model output}

Our evolutionary synthesis code predicts a large variety of observable and related quantities derived from the detailed synthetic spectra. Here we focus on mostly on quantities describing the spectrum in the Lyman continuum, the UV (rest-frame) spectrum, as well as the Ly $\alpha$ and He II $\lambda 1640$ emission lines. The full set of model results, including also numerous other quantities not discussed in this paper, are available in electronic format upon request to one of the authors (DS), on http://obswww . unige.ch/sfr, or via the CDS.

\subsection{Photoionization models}

To predict more accurately the nebular emission from starbursts and to investigate possible departures from the simplified assumptions made in our synthesis models we use the photoionization code CLOUDY version 08 (Ferland et al. 1998). The models we consider are ionization-bounded with a closed, spherical geometry and constant density. These assumptions imply in particular that all ionizing photons are absorbed in the $\mathrm{H}$ II region, i.e. $f_{\mathrm{esc}}=0$. In certain circumstances, especially in high redshift galaxies, a fraction of the Lyman continuum photons are expected to escape (see e.g. Gnedin et al. 2008; Wise \& Cen 2009; Razoumov \& Sommer-Larsen 2009). To first order the results obtained in this paper can simply be rescaled to such cases, as discussed below. The main input parameters of our models are: the spectral energy distribution (SED) of the ionizing source, the nebular density (given by $n_{\mathrm{H}}$ ), the hydrogen number density), the ionization parameter $(U)$, and the nebular metallicity $\left(\mathrm{Z}_{\mathrm{neb}}\right)$. For the SED we adopt black-body spectra described by $T_{\mathrm{bb}}$ and SEDs from our evolutionary synthesis models. The ionization parameter (at the inner edge of the cloud) is defined as:

$U=\frac{Q(\mathrm{H})}{4 \pi r_{\text {in }}^{2} \times n(\mathrm{H}) \times c}$

where $r_{\text {in }}$ is the inner radius of the nebula which has been kept constant in our models $\left(10^{17} \mathrm{~cm}\right)$ and $c$ is the speed of light. The small inner radius used in our calculations results in a sphere-like (not shell-like) geometry of the nebulae. The ionization parameter can change throughout the nebula (decrease towards outer parts).

First, we have calculated the grid of photoionization models using the photoionization code CLOUDY covering $\log (U)=$ $-4,-3,-2,-1 ; T_{\mathrm{bb}}=40000-150000 \mathrm{~K} ; \log (n(\mathrm{H}))=1,2,3$, 

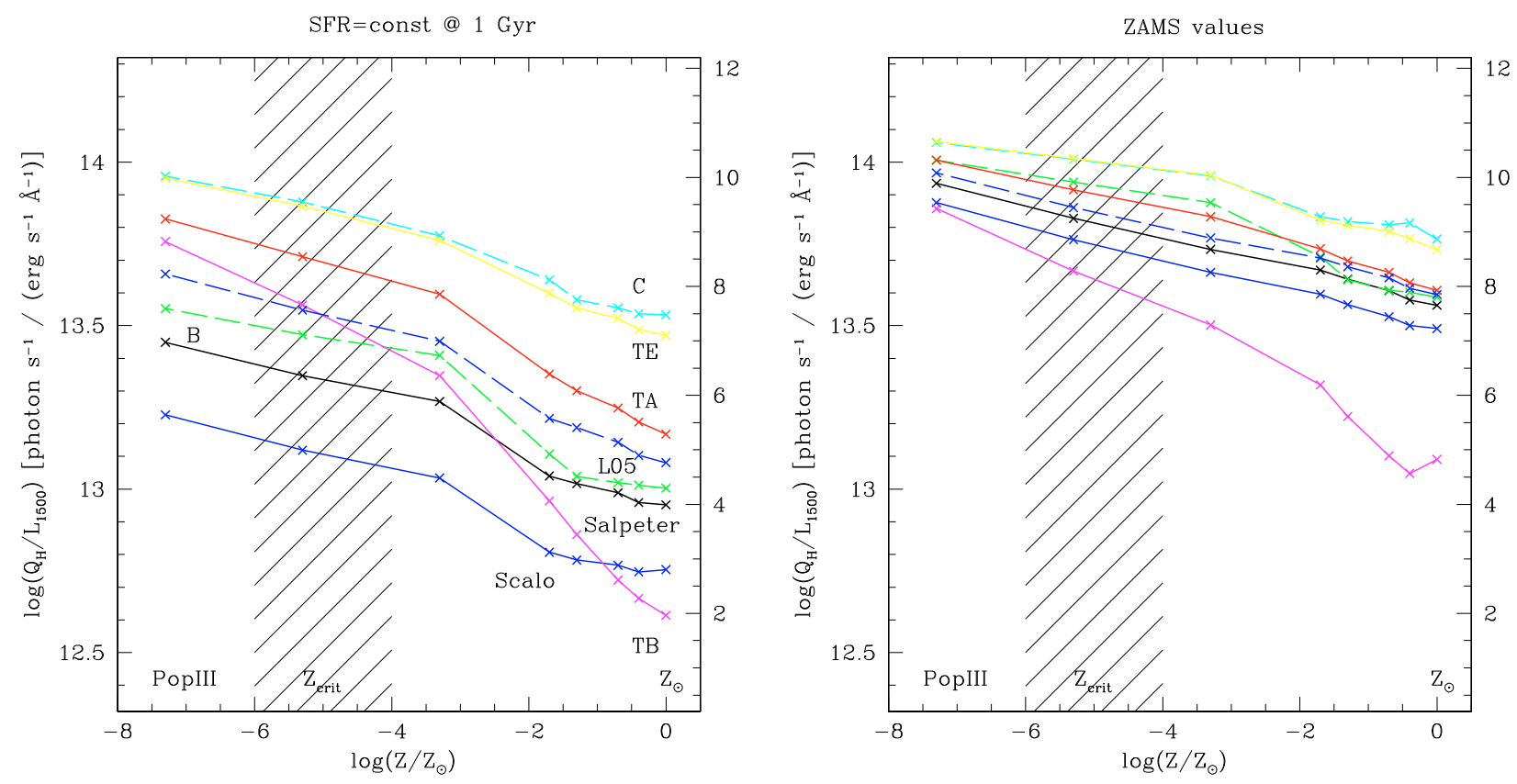

Fig. 1. Relative output of hydrogen ionizing photons to UV continuum light, measured at $1500 \AA$ restframe, $Q_{\mathrm{H}} / L_{1500}$, as a function of metallicity for constant star formation over $1 \mathrm{Gyr}$ (left panel) and very young bursts (right panel). $Q_{\mathrm{H}} / L_{1500}$ is given in $L_{\lambda}$ units on the left side of each panel, and in $L_{v}$ units on the right. Results for different IMFs are shown using the colour codes and labels summarised in Table 1. The shaded area indicates the critical metallicity range where the IMF is expected to change from a "normal" Salpeter-like regime to a more massive IMF (see text).

$4 \mathrm{~cm}^{-3}$ for a primordial nebula and a number of higher metallicities. In total 192 models were computed for each metallicity. In order to keep the same ionization parameter while changing the density of the gas, we adapt the number of ionizing photons (keeping the shape of the SED). The metallicity is defined by scaling the solar abundance pattern.

Note that the electron temperature is neither constant nor fixed in our models. Its spatial distribution results from the computation of each photoionization model and it is a function of depth in the nebula. In practice it depends on all the parameters that are being investigated $\left(T_{\mathrm{bb}}, n_{\mathrm{H}}, U, Z_{\mathrm{neb}}\right)$. For the coolest primordial model (the coolest black body, the lowest density and the lowest ionization parameter) the temperature in the inner part of the cloud is around $12000 \mathrm{~K}$ and for the hottest ones it reaches $\sim 38000 \mathrm{~K}$.

Selected models were subsequently computed using the SEDs from the synthesis models described above.

\section{Predicted UV properties from synthesis models}

We now present and discuss one-by-one the main predictions of our synthesis models for different IMFs (see Table 1), for metallicities from zero (Pop III) to solar, and for two different limiting star-formation histories (bursts and $S F R=$ const.). Since properties of stars below $Z \lesssim 10^{-9}$ (i.e. $Z / Z_{\odot} \lesssim 10^{-7.3}$ ) essentially converge to those of metal-free stars we assign this metallicity value to Pop III stars, as in Schaerer (2003).

Note that all UV continuum predictions from the synthesis models described in this Section are based on the simplified assumptions spelled out above to compute nebular emission. This implies in particular that at low metallicities the contribution from the two-photon continuum process should be higher, increasing thus e.g. the predicted UV luminosity at $1500 \AA$, for the reasons discussed in Sect. 4.

\subsection{Ionizing photon production}

A quantity of interest, e.g. to determine the contribution of galaxies to cosmic reionization, is the the relative output of hydrogen ionizing photons to observable UV light. Here we provide $Q_{\mathrm{H}} / L_{1500}$, where the Lyman continuum flux $Q_{\mathrm{H}}$ is expressed in units of photon $\mathrm{s}^{-1}$, and the UV restframe luminosity at $1500 \AA$ is $L_{1500}$ in $L_{\lambda}\left(\mathrm{erg} \mathrm{s}^{-1} \AA^{-1}\right)$ or $L_{v}\left(\mathrm{erg} \mathrm{s}^{-1} \mathrm{~Hz}^{-1}\right)$ units ${ }^{1}$. Alternatively, to derive the Lyman continuum output per unit stellar mass (or per baryon), $Q_{\mathrm{H}} / L_{1500}$ discussed here can be combined with the ratio $S F R / L_{1500}$ given below, or can directly be derived from the available data files.

In Fig. 1 we show $Q_{\mathrm{H}} / L_{1500}$ as a function of metallicity for constant star formation over $1 \mathrm{Gyr}$ (CSFR, left panel), and very young ( $\$ 0-4 \mathrm{Myr}$ ) populations (right panel). As expected $Q_{\mathrm{H}} / L_{1500}$ increases with decreasing metallicity, since the ionizing flux depends very strongly on the effective stellar temperature and hence increases more rapidly than the UV luminosity. The IMF dependence also behaves as expected, with the IMFs favouring the most massive stars showing also the highest the $Q_{\mathrm{H}} / L_{1500}$ ratios, since $Q_{\mathrm{H}}$ increases more rapidly with stellar mass than the UV luminosity. Notable is actually the increase of $Q_{\mathrm{H}} / L_{1500}$ from $Z_{\odot}$ to $\sim 10^{-4} Z_{\odot}$, where no major change of the IMF is expected (and a Salpeter IMF is favoured). For CSFR and for a fixed IMF, the increase of the relative ionizing power from solar metallicity to Pop III is typically $\sim 0.4-0.5$ dex, i.e. a factor 2 to 3 . When considering an IMF change from Salpeter to a massive IMF (i.e. all cases except Salpeter and Scalo) the increase of $Q_{\mathrm{H}} / L_{1500}$ is larger, approximately 0.6 to 1 dex between solar and zero metallicity. Only for the "TB" IMF, a narrow, lognormal mass function peaked at $M=15 M_{\odot}$, we find a more extreme dependence on metallicity. This is precisely due to the fact that this IMF singles out a narrow mass range, instead of

${ }_{1}$ The transformation is $\log \left(Q_{\mathrm{H}} / L_{\lambda}(1500 \AA)\right)=\log \left(Q_{\mathrm{H}} / L_{v}(1500 \AA)\right)$ 12.12 . 
time evolution for bursts/CSFR models and different IMFs

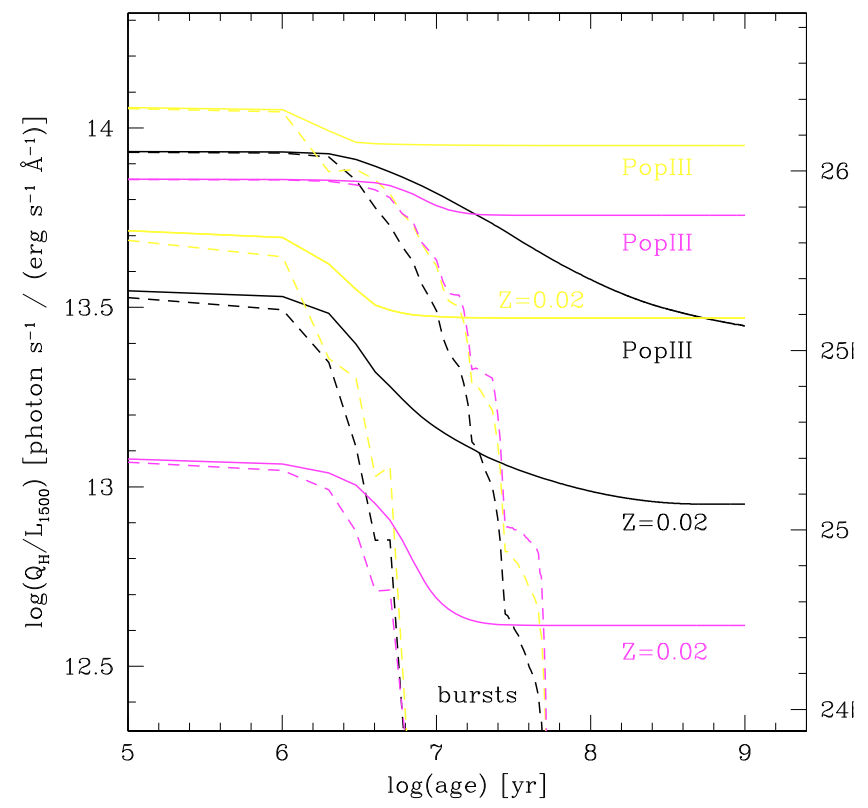

Fig. 2. Temporal evolution of $Q_{\mathrm{H}} / L_{1500}$ for selected IMFs (Salpeter, TB, TE, colour-coded as in previous figures) and metallicities (Pop III, $Z_{\odot}$, labeled). The solid curves show the time evolution for constant SFR models towards their equilibrium value, the dashed curves instantaneous burst models. See text for discussion.

averaging the metallicity dependence of stellar properties over a larger interval in mass.

The right panel of Fig. 1 shows a narrower range of $Q_{\mathrm{H}} / L_{1500}$ for zero age or very young ( $\$ 0-4 \mathrm{Myr})$ populations. This is natural, since in this case no "average" is made over populations of very different stellar ages and hence over strong variations of stellar parameters. More important is the fact that higher values of $Q_{\mathrm{H}} / L_{1500}$ are obtained for young populations. This is mostly due to the fact that such populations emit a lower UV luminosity per unit SFR since a longer timescale is needed to reach the "equilibrium value" of the UV output (cf. below). The ZAMS values shown here correspond to the maximum of $Q_{\mathrm{H}} / L_{1500}$ expected for stellar populations of different ages and SF histories.

To illustrate this dependence on the SF timescale, $Q_{\mathrm{H}} /$ $L_{1500}(t)$ is shown in Fig. 2 for selected IMFs and metallicities. These curves show the smooth transition from the predicted "ZAMS" to the CSFR values over timescales from $\sim 10^{7} \mathrm{yr}$ for massive IMFs (e.g. TE, TB) to $\sim 0.4-1$ Gyr for the Salpeter IMF. Note also that the timescale for UV properties to reach equilibrium increases with decreasing metallicity, due to the higher effective temperatures on the ZAMS at low $Z$. In short, we caution that the relative ionizing photon to UV ratio $Q_{\mathrm{H}} / L_{1500}$ may be uncertain by a factor of $\sim 4$ depending on the SF timescale (for constant SF), or even more for bursts.

Finally, it should be noted that at low metallicity the contribution of the two-photon continuum may be larger, as shown later in Sect. 4, leading to somewhat lower values of $Q_{\mathrm{H}} / L_{1500}$. For constant SF this amounts to a decrease of $Q_{\mathrm{H}} / L_{1500}$ by $\sim 0.2$ dex for Pop III and the most extreme IMFs (TE, C), and smaller changes otherwise. For zero metallicity populations on the ZAMS $Q_{\mathrm{H}} / L_{1500}$ should be reduced by $\sim 0.15-0.3$ dex for all IMFs, and less at higher metallicity.

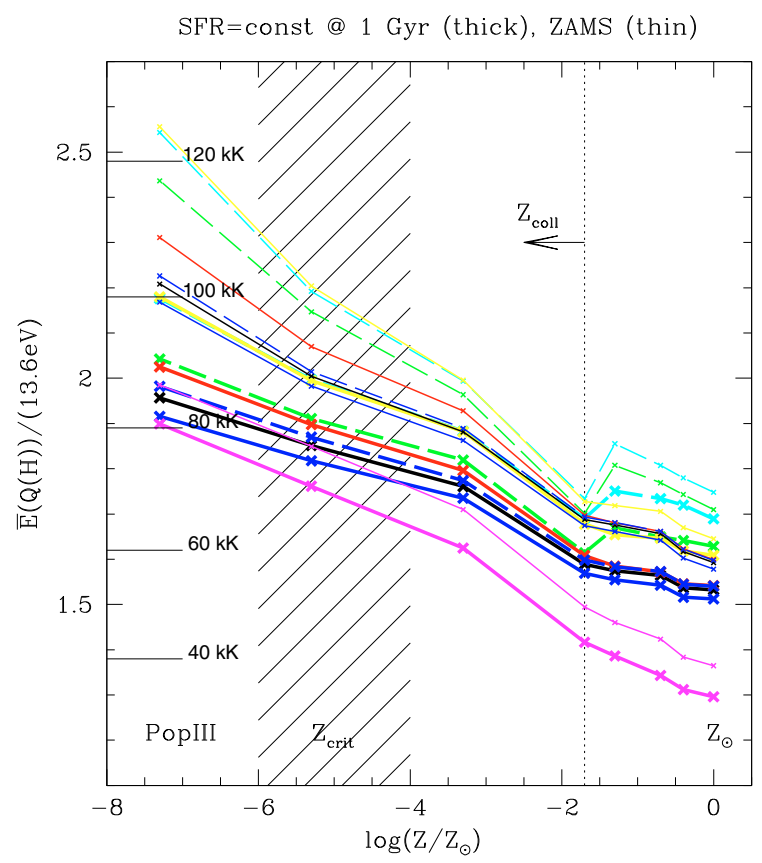

Fig. 3. Mean ionizing photon energy in units of $13.6 \mathrm{eV}$ as a function of metallicity, shown for $S F R=$ const. (thick lines) and for the ZAMS (thin lines). Results for different IMFs are shown using the same colour codes as in Fig. 1 (cf. Table 1). Lined and labels on the left indicate the corresponding blackbody temperatures. $Z_{\text {coll }}$ shows the approximate metallicity limit below with collisional effects lead to significant departures from case B.

\subsection{Properties of the ionizing spectra}

The properties of the ionizing spectra, such as their hardness, detailed shape and others have already been discussed in Schaerer (2003) and shall not be repeated here. For example, the hardness $Q\left(\mathrm{He}^{+}\right) / Q(\mathrm{H})$, measured by the ratio of $\mathrm{He}^{+}(>54 \mathrm{eV})$ to hydrogen ionizing $(>13.6 \mathrm{eV})$ photons, we predict from our new models are already bracketed by the the values predicted in Schaerer (2003, see their Fig. 5) for the Salpeter and the "C" IMF.

An interesting quantity describing the ionizing spectra is the average energy of the photons emitted in the Lyman continuum $\bar{E}(Q(\mathrm{H}))$ (see definition in Eq. (6)). This quantity and its dependence on metallicity and IMF is plotted in Fig. 3 for constant SF (thick lines) and for the ZAMS (thin lines). The corresponding blackbody temperatures $T_{\mathrm{bb}}$ with the same mean ionizing photon energy are also shown for illustration. Typically $\bar{E}(Q(\mathrm{H}))$ is found to $\sim 1.5-2.5$ times $13.6 \mathrm{eV}$, the ionizing potential of neutral hydrogen, and its behaviour with IMF, metallicity, and age behaves as expected. These values correspond to a range of blackbody temperatures from $\sim 50$ to $120 \mathrm{kK}$ for the hardest spectra.

In this figure we also indicate the approximate metallicity limit $Z_{\text {coll }}$, below which collisional effects lead to significant departures from case $\mathrm{B}$, as shown below. In this metallicity range $\bar{E}(Q(\mathrm{H}))$ can also be used to compute more accurately the intrinsic Ly $\alpha$ emission line strength (see Eq. (8)).

\subsection{SFR calibrations from the UV continuum}

Figure 4 illustrates the variation of the UV luminosity for CSFR as a function of metallicity and for the different IMFs. Plotted is the conversion factor $c$, defined by $S F R=c \times L_{v}$, where 


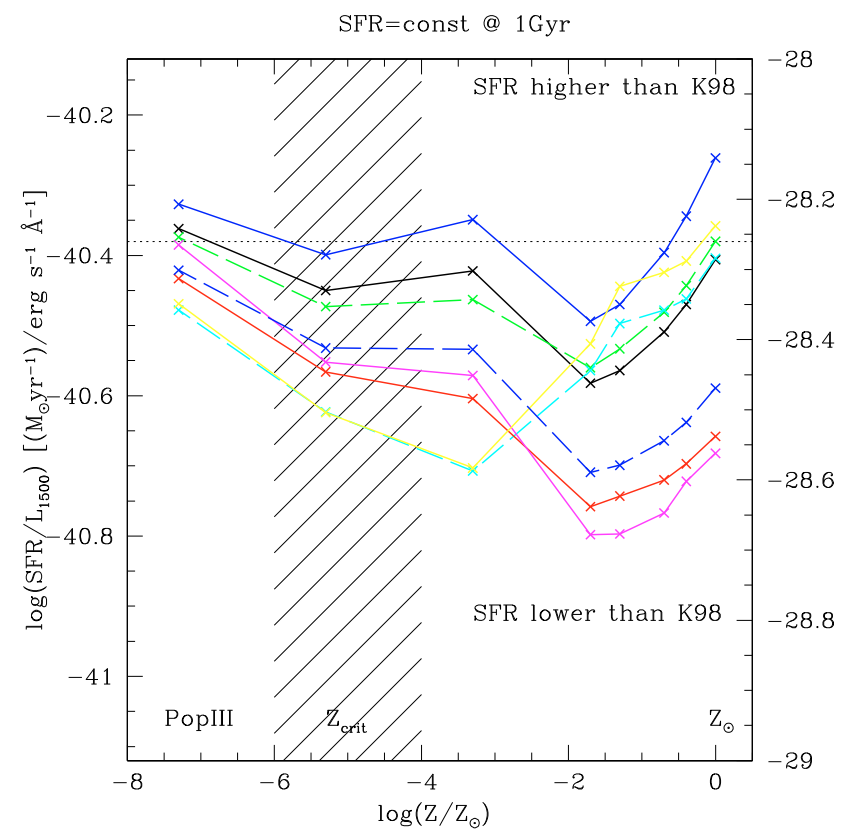

Fig. 4. Dependence of the SFR(UV) calibration on metallicity and IMF. Shown is the SFR per unit UV luminosity at $1500 \AA$ in units of $M_{\odot} \mathrm{yr}^{-1}$ per $\left(\mathrm{erg} \mathrm{s}^{-1} \AA^{-1}\right)$ on the left $y$-axis, or in $M_{\odot} \mathrm{yr}^{-1}$ per $\left(\mathrm{erg} \mathrm{s}^{-1} \mathrm{~Hz}^{-1}\right)$ on the right $y$-axis. Same symbols and colours as in previous figures (cf. Table 1). These values assume constant SF over 1 Gyr. The dotted horizontal line show the value of the Kennicutt (1998) SFR(UV) calibration rescaled to a Salpeter IMF with $M_{\text {low }}=1 M_{\odot}$ for comparison. Above/below this line the SFR deduced from the UV luminosity is higher/lower.

$L_{v}$ is the UV luminosity in units of $\operatorname{erg~s}^{-1} \mathrm{~Hz}^{-1}$, and SFR is the star formation rate in $M_{\odot} \mathrm{yr}^{-1}$. As expected our model with Salpeter IMF agrees well with the widely used calibration from Kennicutt (1998) at $Z_{\odot}$ after rescaling the latter by a factor 2.55 to account for our adopted value for the lower mass cut-off $\left(M_{\text {low }}=1 M_{\odot}\right)^{2}$. This figure clearly shows that in most cases the use of the Kennicutt calibration at low metallicity may overestimate the SFR, given the higher intrinsic UV output of such stellar populations. However, this may not be realistic since it relies on the assumption of CSFR over a long timescale $\left(\gtrsim 10^{8.3 \ldots 9} \mathrm{yr}\right)$. In younger populations the UV luminosity per unit $S F R$ is lower, and hence $c$ and the determined SFR higher (e.g. Schaerer 2000).

In fact, the non-monotonous behaviour of $S F R / L_{1500}$ with metallicity observed in Fig. 4 is due to the dependence of the stellar contribution to the total UV output at this wavelength. Indeed, the stellar UV output increases with decreasing $Z$ down to $\sim 1 / 50 Z / Z_{\odot}$, due to the decrease of the average temperature of stars over their lifetime. At even lower metallicities, however, their UV output (per unit SFR) decreases since the bulk of their flux is emitted at $\ll 1500 \AA$ (cf. Fig. 2 in Schaerer 2003). This implies, for a fixed IMF, a re-increase of $S F R / L_{1500}$ at $Z / Z_{\odot} \lesssim 1 / 50$, which is only somewhat moderated by the increasing nebular contribution. Indeed, the latter contributes typically $\sim 10-40 \%$ of the flux at $1500 \AA$ at SF equilibrium (see Fig. 8) In other words neglecting the nebular continuum would lead to differences of $\sim 0.05-0.15$ dex in the $S F R$ calibrations. At low metallicity the contribution of the two-photon continuum

\footnotetext{
2 More explicitely the figure shows $\log c$, where $\log S F R=\log c+$ $\log L_{1500}+\log c_{M}$, and $c_{M}=2.55$ for the IMF adopted by Kennicutt $\left(M_{\text {low }}=0.1 M_{\odot}\right)$, or $c_{M}=1$ for $M_{\text {low }}=1 M_{\odot}$.
}

may be larger, as shown in Sect. 4, leading to somewhat lower values of $S F R / L_{1500}$ than shown in Fig. 4. For Pop III and the most extreme IMFs (TE, C) this implies a downward revision of $\sim 0.2$ dex.

\subsection{Predicted Ly $\alpha$ emission}

The Ly $\alpha$ equivalent widths predicted by our standard models (using Eq. (2)) for all IMFs and metallicities are shown with thin lines in Fig. 5 for constant star-formation (left panel) and for the ZAMS (right), the latter representing the maximum of $W(\operatorname{Ly} \alpha)$ for each IMF. The dependences of $W(\operatorname{Ly} \alpha)$ are as expected from $Q_{\mathrm{H}} / L_{1500}$ and the values shown here bracket those already presented in Schaerer (2003) (with more limited variations of the IMF). To illustrate the departure from case B found at low metallicity from photoionization models (see Sect. 4), we also show $W(\operatorname{Ly} \alpha)$ computed from Eq. (4) in the low density regime (i.e. for $\tilde{f}_{\text {coll }}=2 / 3$, thick lines). Here, the equivalent widths are a factor $\sim 1.5-2.5$ higher than our "standard" predictions (cf. Fig. 3), since we assume that the continuum close to Ly $\alpha$ is unchanged by this departure from case B.

A few words of caution about $W(\operatorname{Ly} \alpha)$ are appropriate. First, note that for our computations of $W(\operatorname{Ly} \alpha)$ we use the continuum flux at $1215.67 \AA$ obtained from linear interpolation of the total (stellar + nebular) continuum (in log) between 1190 and $1240 \AA$, chosen to avoid underlying stellar Ly $\alpha$ absorption and other absorption lines if present (cf. above). While $W(\mathrm{Ly} \alpha)$ is well defined theoretically, comparisons with observations require some caution, given the possible complexity of the continuous spectrum shortward (due to the IGM in particular) and longward of Ly $\alpha$ (due to non-monotonic shape of the nebular continuum), and given different choices of broadband filters (see e.g. the simulations of Hayes \& Östlin 2006).

How much of the total radiative energy from the starburst is emitted in the Ly $\alpha$ line? For constant star-formation the fraction of the $\operatorname{Ly} \alpha$ to the bolometric luminosity, $L(\operatorname{Ly} \alpha) / L_{\mathrm{bol}}$, is shown in Fig. 6 for all metallicities and IMFs. At solar metallicity and for a Salpeter IMF we obtain the well-known estimate of $L(\operatorname{Ly} \alpha) / L_{\mathrm{bol}} \sim 3-6 \%$ found in the first papers promoting Ly $\alpha$ searches at high redshift (e.g. Partridge \& Peebles 1967). The fraction of luminosity emitted in Ly $\alpha$ increases with decreasing metallicity, due to the higher ionizing photon flux output per unit stellar mass. When case B departures at low metallicity are taken into account, we find that $L(\operatorname{Ly} \alpha) / L_{\text {bol }}$ can reach up to $\sim 20-40 \%$ depending on the IMF, i.e. up to 10 times more than expected from earlier calculations! The highest values are comparable to those from the photoionization models of Panagia (2002) using very hot black body spectra. For younger populations $L(\operatorname{Ly} \alpha) / L_{\mathrm{bol}}$ is less dependent on the IMF than for $S F R=$ const. shown here; values of $L(\operatorname{Ly} \alpha) / L_{\text {bol }} \sim 0.15-0.20$ (0.35-0.40) are obtained at low $Z$ with our standard (departure from case B) assumptions.

\subsection{He II line emission from very metal-poor starburst galaxies}

Our standard predictions for He II $\lambda 1640$ (using Eq. (3)) for constant star-formation and young bursts are shown in Fig. 7. They complement our earlier predictions in Schaerer (2003), and show the expected behaviour. Clearly, strong nebular He II $\lambda 1640$ emission from starbursts is only expected at very low metallicity and for IMFs producing enough massive stars. As we will show below, the predicted intensity of He II $\lambda 1640$ (and 

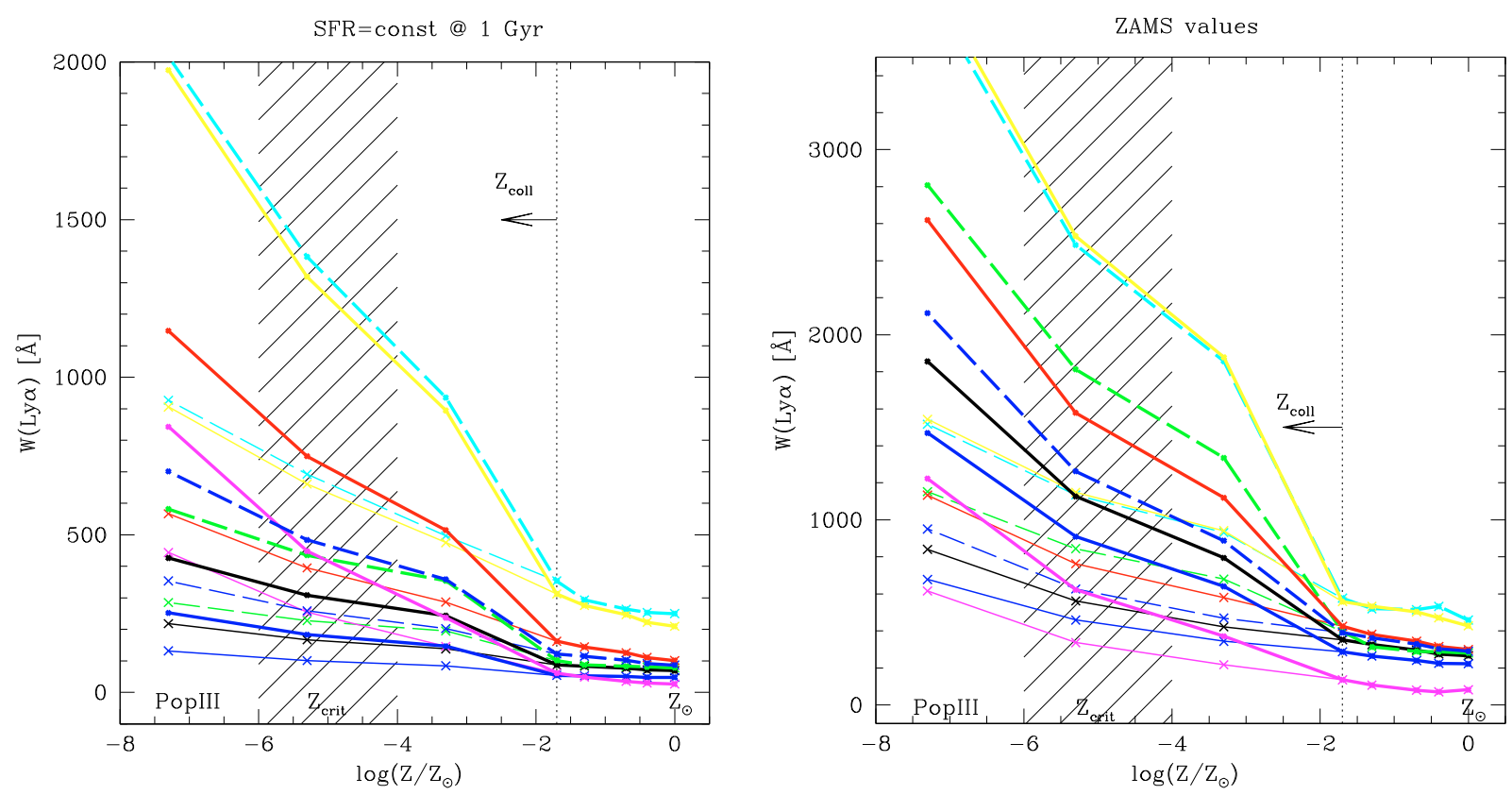

Fig. 5. Predicted Ly $\alpha$ equivalent width as a function of metallicity for constant star formation (left panel) and very young ( $\leq 1-2 \mathrm{Myr}$ ) bursts (right panel). Note the different vertical scales on the two plots. Thin lines show our "standard" predictions, thick lines the predicted $W($ Ly $\alpha)$ accounting to first order for departure from case B following Eq. (8) (assuming low density, i.e. $\tilde{f}_{\text {coll }}=2 / 3$, and neglecting the increase of the two-photon continuum), leading to an increase by up to a factor $\sim 1.5-2.5$ at low metallicities $\left(Z \lesssim Z_{\text {coll }}\right)$. Results for different IMFs are shown using the same colour codes as in Fig. 1 (cf. Table 1).

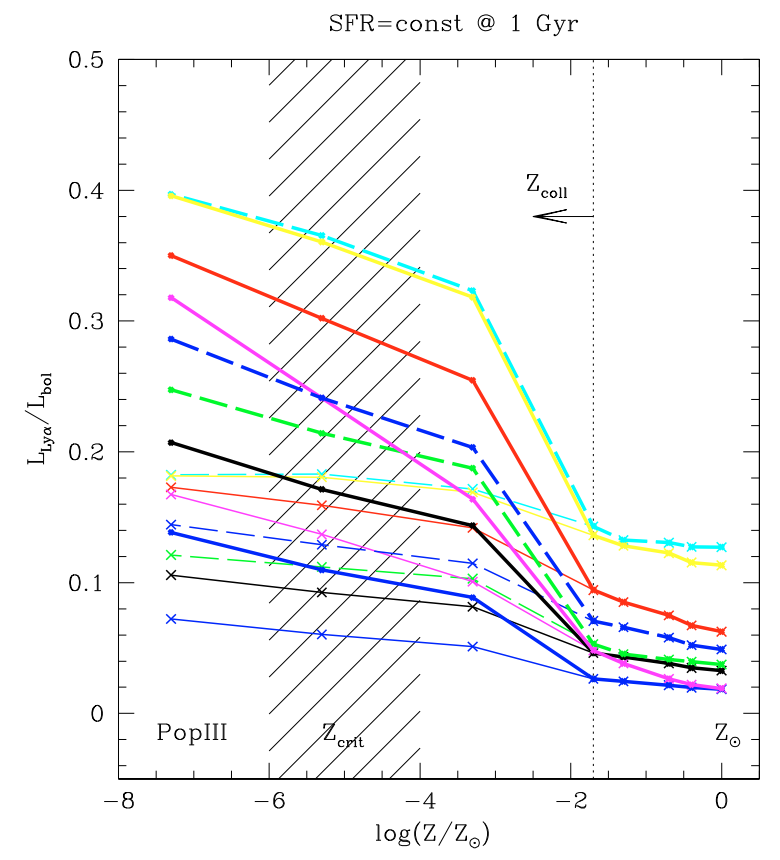

Fig. 6. Fraction of the Ly $\alpha$ luminosity to the total bolometric luminosity, $L(\operatorname{Ly} \alpha) / L_{\mathrm{bol}}$ for $S F R=$ const. as a function of metallicity and IMF. Results for different IMFs are shown using the same colour codes as in Fig. 1 (cf. Table 1). Thin lines show results using our "standard" Ly $\alpha$ predictions; thick lines the improved results accounting for departures from case $B$ at very low metallicity. Note the resulting strong increase of our revised $L(\operatorname{Ly} \alpha) / L_{\mathrm{bol}}$ values from solar to very low metallicity!

other $\mathrm{He}^{+}$recombination lines) depends, however, also on the ionization parameter and on the ISM density to some extent. Complete photoionization models predict generally fainter He II $\lambda 1640$ emission, as discussed in Sect. 4.

\subsection{Importance of the nebular continuum}

Figure 8 shows the contribution of nebular continuous emission to the total UV light at $1500 \AA$ for all metallicities, IMFs, and for the usual limiting cases of star-formation histories. Whereas for "normal" metallicities and IMFs the contribution is relatively small ( $\$ 5 \%$ for $S F R=$ const.), the importance of the nebular continuum is larger for young bursts and/or low metallicity, as already stressed by Schaerer (2002) and Schaerer (2003).

Since the nebular continuum at $1500 \AA$ is generally dominated by the two-photon continuous emission process, departures from Case B will lead to stronger nebular emission than shown here at very low metallicity (see Sect. 4). To first order, the total two-photon emission is then enhanced by a factor $P$ for low ISM densities, increasing thus the contribution of the nebular continuum to the total (stellar + nebular) emission. At high density, the two-photon emission tends to zero. However, since the shape of the nebular continuum depends on the detailed conditions in the nebula (density, temperature, etc.) which are not constant as assumed in the synthesis models, it is not possible to predict more accurately how departures from case B affects the nebular continuum, without resort to photoionization models.

\subsection{Predicted UV slope}

From our synthesis models we also measured the slope of the UV continuum with and without nebular emission (cf. Schaerer $\&$ Pelló 2005). In Fig. 9 we show a condensed overview of various $\beta$-slopes for very young populations (ZAMS), representing the steepest slopes, i.e. the minimum for $\beta$, predicted from models. As before, the predictions are shown for all IMFs and metallicities. Thick (thin) lines show $\beta_{1550}\left(\beta_{2000}\right)$, defined as the slope between 1300-1800 (1800-2200) ^ respectively ${ }^{3}$. The upper set of lines shows the $\beta$ slopes of the total spectrum, including

${ }^{3}$ We use the standard definition $F_{\lambda} \propto \lambda^{\beta}$. 

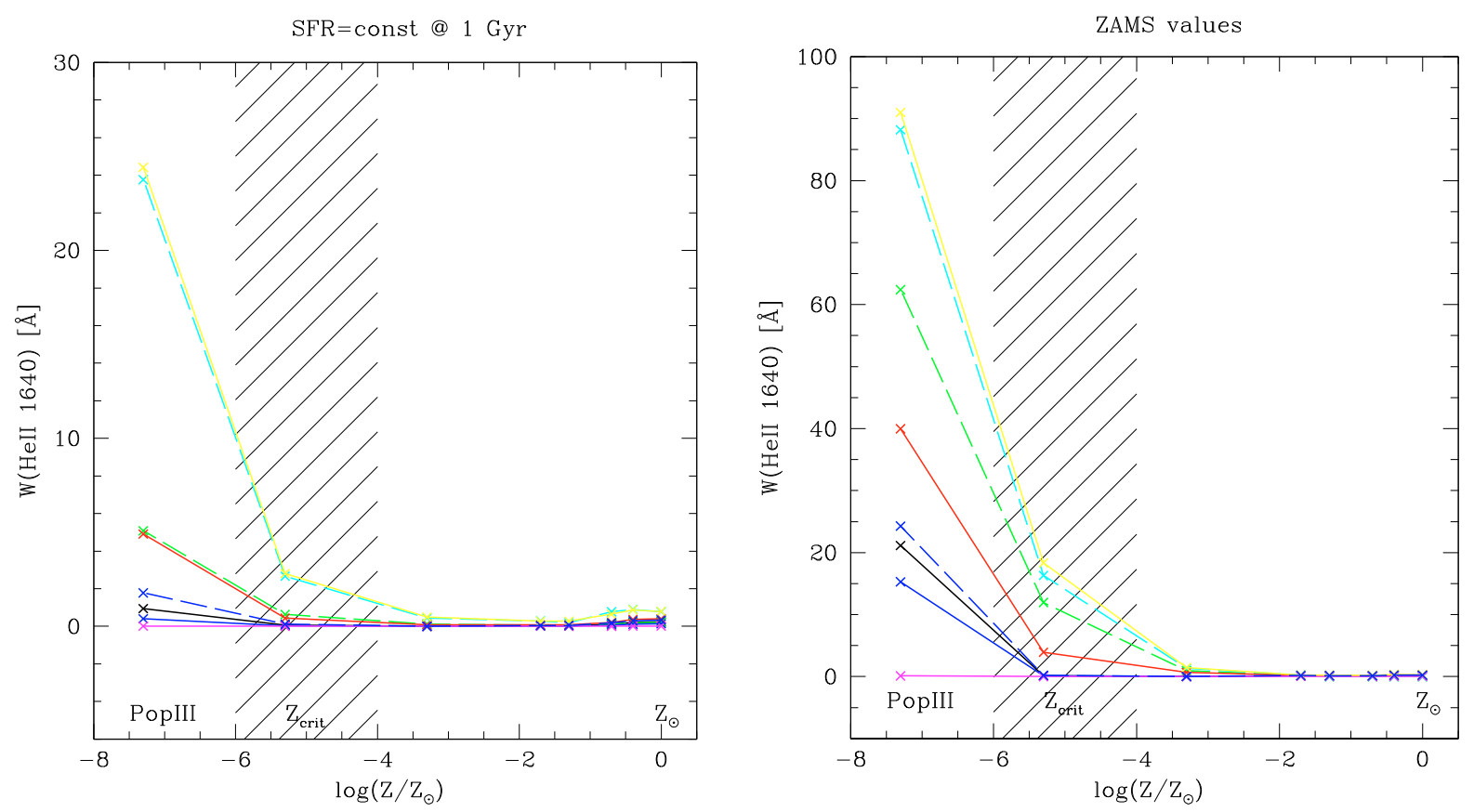

Fig. 7. Predicted He II $\lambda 1640$ equivalent width as a function of metallicity for constant star formation (left panel) and very young ( $\leq 1-2$ Myr) bursts (right panel). Note the different vertical scales on the two plots. Results for different IMFs are shown using the same colour codes as in Fig. 1 (cf. Table 1). Note that photoionization models predict generally fainter He II $\lambda 1640$ emission, hence lower equivalent widths, except for high ISM densities (see Sect. 4.3).
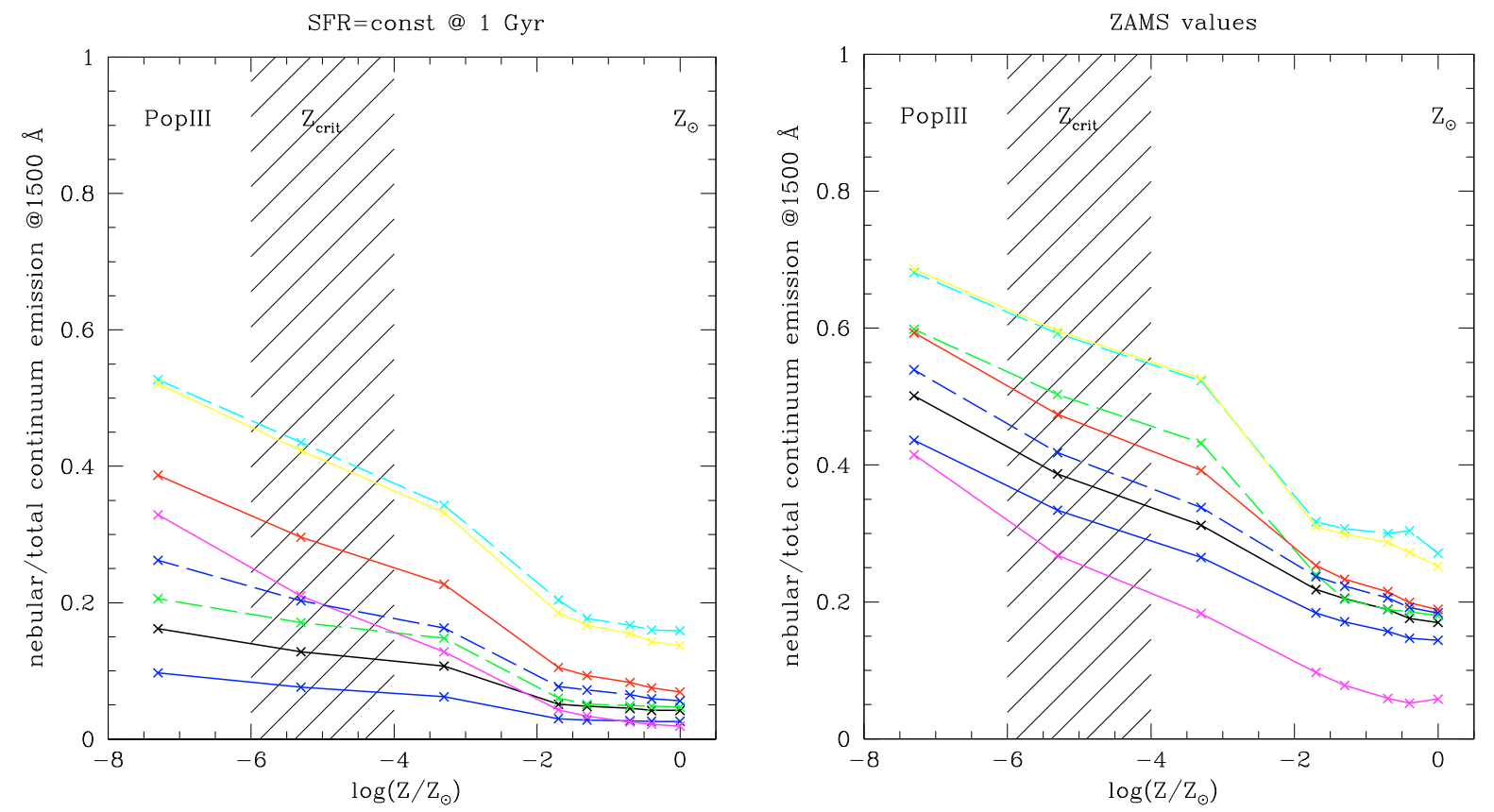

Fig. 8. Contribution of nebular continuum emission to the total emission in the restframe UV at $1500 \AA$ as derived from our evolutionary synthesis models. Shown are model for constant star formation (left panel) and very young ( $\leq 1-2 \mathrm{Myr}$ ) bursts (right panel). Results for different IMFs are shown using the same colour codes as in Fig. 1 (cf. Table 1).

stellar and nebular continuum, the lower lines using the pure stellar spectrum.

Clearly, the UV slope is strongly affected by nebular emission, leading to a significant flattening of the spectrum (cf. Figs. 2 in Schaerer 2003). While the stellar SED becomes steeper with decreasing metallicity, the total spectrum exhibits the opposite behaviour. If we assume a varying contribution of the nebular continuum we may obtain any intermediate value of $\beta$ between the "stellar+nebular" and "pure stellar" cases. This fact and the dependence of $\beta$ on the star-formation history and age (see e.g. Fig. 1 in Schaerer \& Pelló 2005), shows that the UV slope cannot be used to determine metallicity. For constant star-formation (not shown here), the bulk of the models show equilibrium values of $\beta \sim-2.6$ to -2 , quite independently of metallicity and IMF.

Figure 9 also shows a difference of the order of $0.2-0.3$ between $\beta_{1500}$ and $\beta_{2000}$. Such differences may be relevant for comparisons of the UV slope estimated from broad-band filters. Last, 
A. Raiter et al.: UV properties of starburst galaxies
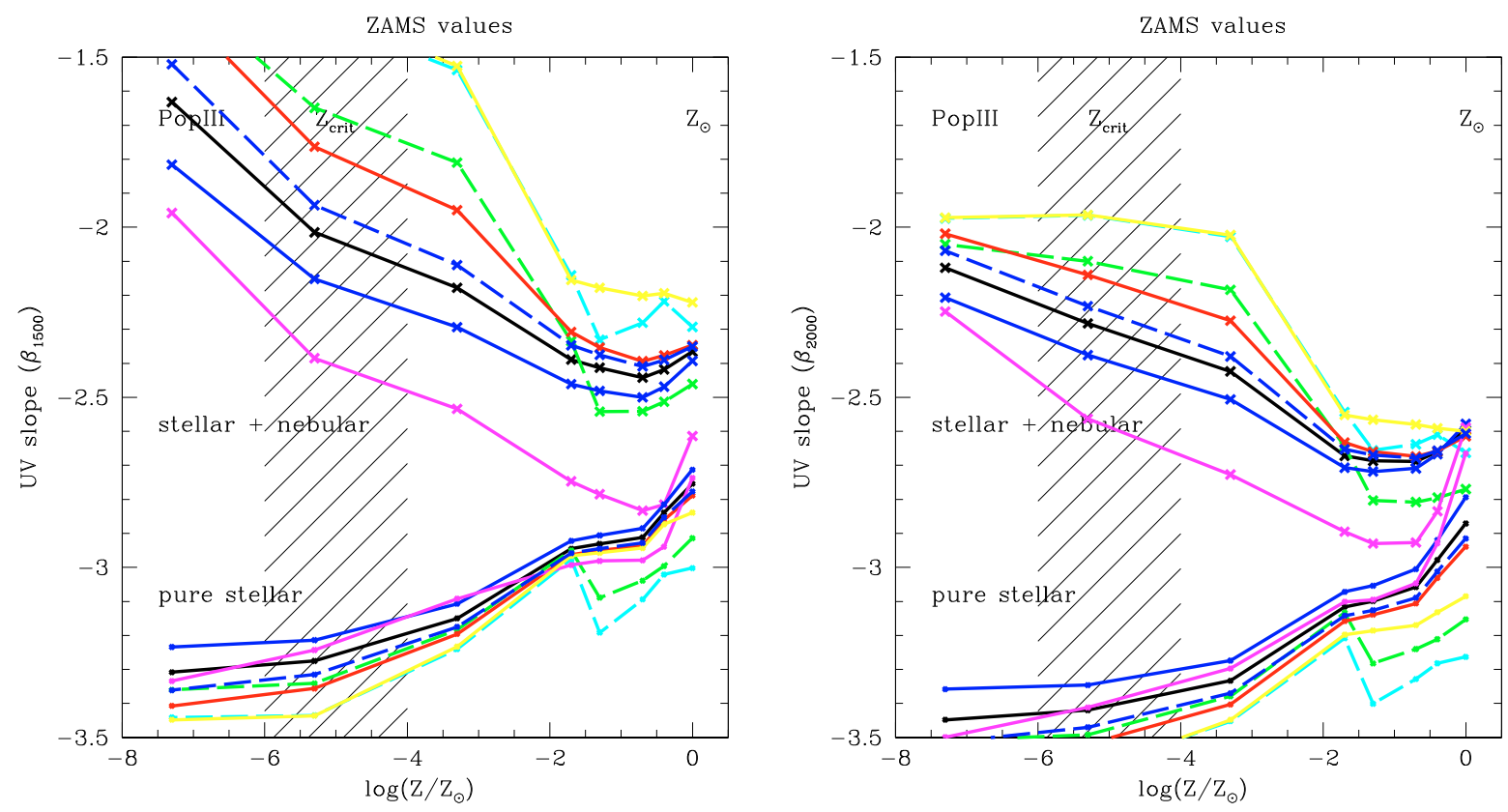

Fig. 9. Left: predicted UV slope $\beta_{1500}$ and for all IMFs and metallicities. The values are shown for very young (ZAMS) populations, which correspond to the bluest possible slopes (i.e. minimal $\beta$ values). The upper set of lines shows the UV slopes of the total spectrum (stellar + nebular continuum), the lower lines using the pure stellar spectrum. Results for different IMFs are shown using the same colour codes as in Fig. 1 (cf. Table 1). Right: same as the left panel, for $\beta_{2000}$. Note the difference between $\beta_{1500}$ and $\beta_{2000}$, indicative of the deviation of the true SED from a simple power law.

but not least, the precise shape of the nebular continuum is determined by the detailed nebular structure (i.e. its detailed temperature and density structure) and can be affected by departures from case B, as the case of the two-photon continuum discussed in depth below.

\section{Nebular predictions using photoionization models}

For low metallicity nebulae ionized by very hot stars, the conventional case B predictions for line and continuum emission are not good approximations to the appropriate nebular astrophysics. In this Section we present the predictions of detailed photoionization modeling for such nebulae with metallicities ranging from zero (primordial $=$ Pop III) to solar, explain the origin of departures from case $\mathrm{B}$, and present a parameterisation of the results than can be readily employed for the interpretation of low metallicity nebulae. For simplicity, we first use black body spectra as ionizing sources and discuss later how to compare these with stellar SEDs. We have examined the nebular emission as a function of the stellar (black body) temperature $T_{\mathrm{bb}}$, the hydrogen number density $n_{\mathrm{H}}$, the ionization parameter $(U)$, and the nebular metallicity $\left(Z_{\text {neb }}\right)$, using the models described above (Sect. 2.2).

Our analysis will focus mainly on the Ly $\alpha$ line and the associated two-photon continuum emission, on nebular He II emission, and on the global nebular continuum.

\subsection{Lyo line and two-photon continuum emission}

In Fig. 10 we show the deviation of the Ly $\alpha$ (black symbols) and $2 \gamma$ emission (red) and other quantities as predicted from photoionization models with primordial composition and different densities with respect to their case B values. To calculate case B luminosities we adopt Eq. (2) and assume $L(2 \gamma)=0.5 c_{1} Q(\mathrm{H})$ for the luminosity in the two-photon continuum. The numerical factor $0.5 \approx \alpha_{2^{2} \mathrm{~S}}^{\mathrm{eff}} / \alpha_{2^{2} \mathrm{P}}^{\mathrm{eff}}$ is appropriate for low densities. For simplicity we always compare the results to the low density limit case $\mathrm{B}$ predictions.

At low density, the luminosity of both Ly $\alpha$ and $2 \gamma$ emission are increased by a factor of $\sim 1.3$ to 3 over the black body temperature range considered here. The physical reason for this strong departure from case B is due to collisional effects, which increase the population of the $n=2$ level of hydrogen from which additional ionizations can take place, leading overall to an increased ionization rate in the nebula. In equilibrium, this implies an increased recombination rate and higher $\operatorname{Ly} \alpha$ and $2 \gamma$ luminosities. Collisional excitation is significant in (very) low metallicity nebulae with hot ionizing sources because radiative cooling is reduced, leading to higher electron temperatures. Photoionization from excited states (here from $n=2$ ), in particular, is not taken into account under case B assumptions.

To describe quantitatively the effect of enhanced photoionization rates, including ionization from the excited $n=2$ state, it is sufficient to consider the mean energy of the ionizing photons in units of the ionization potential of hydrogen, $P$, given by

$P=\frac{\bar{E}}{13.6 \mathrm{eV}}=\left(\frac{\int_{13.6 \mathrm{eV}}^{\infty} F_{\nu} \mathrm{d} v}{\int_{13.6 \mathrm{eV}}^{\infty} \frac{F_{v}}{h v} \mathrm{~d} v}\right) /(13.6 \mathrm{eV})$,

where $\bar{E}$ is the average ionizing photon energy in the Lyman continuum in units of $\mathrm{eV}$. Indeed, as Fig. 10 shows, the enhancement of the Ly $\alpha$ and two-photon continuum emission, which both measure the effective recombination rate, scales very accurately with $P$ at low density. This scaling shows that the available energy of the Lyman continuum photons - which is in excess of the necessary minimum of $13.6 \mathrm{eV}$ - is "optimally" used to maximise the number of photoionizations, leading to an effective increase of the ionization rate from $\propto Q(\mathrm{H})$ to $\propto P \times Q(\mathrm{H})$, and hence to the same increase in the recombination rates, i.e. also in the $\operatorname{Ly} \alpha$ and $2 \gamma$ luminosities. 

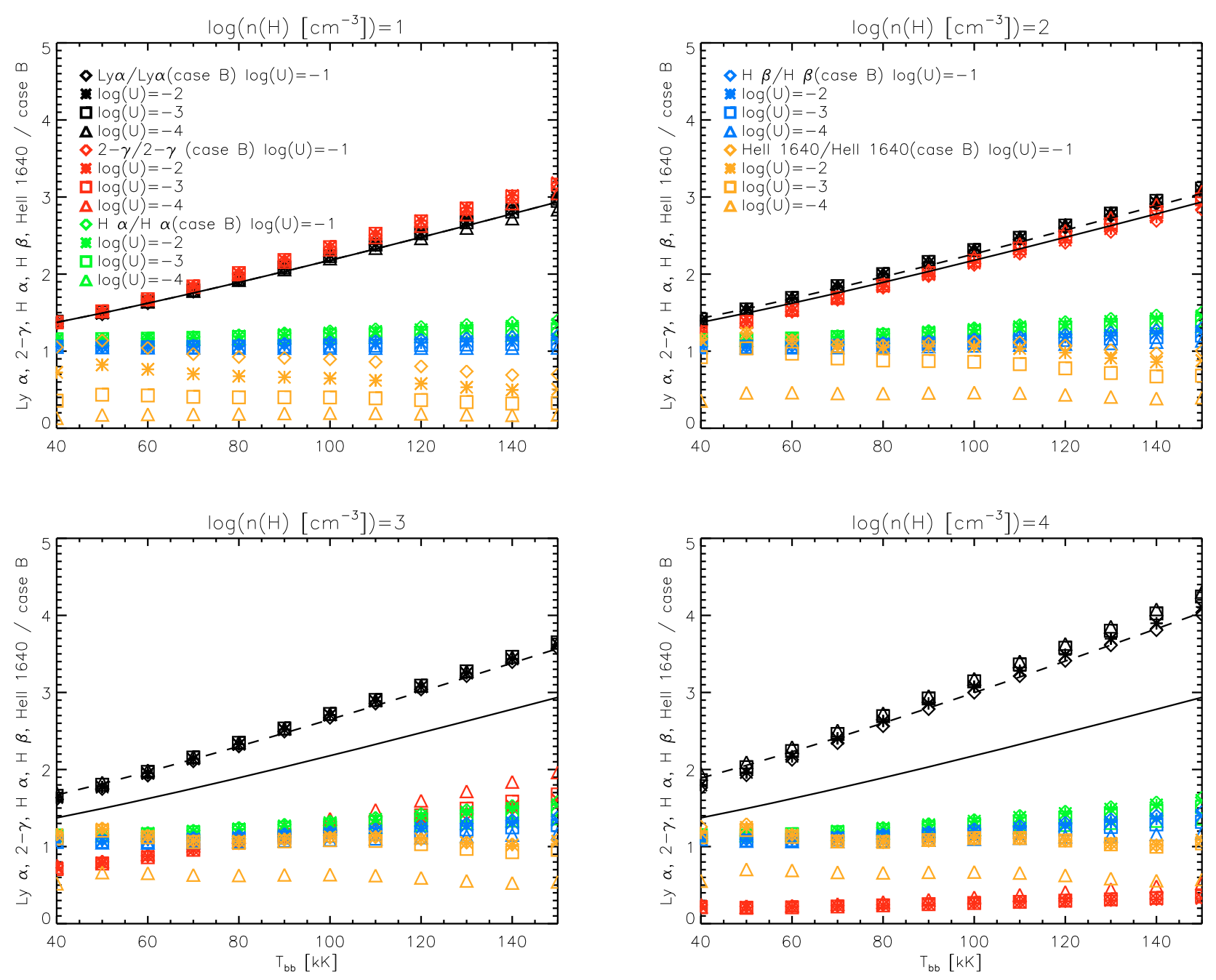

Fig. 10. Ly $\alpha$ (black), $2 \gamma$ (red), $\mathrm{H} \alpha$ (green), $\mathrm{H} \beta$ (blue), He II $1640 \AA$ (orange) luminosities over their predicted case B luminosities for the primordial nebula. The black solid line shows $P$ (Eq. (6)), the dashed lines show $P \times \tilde{f}_{\text {coll }} / \tilde{f}_{\text {coll }}\left(\log \left(n_{\mathrm{H}}=1\right)\right)(\mathrm{cf}$. Eq. (7)). Different symbols represent different ionization parameters, namely open diamonds correspond to $\log (U)=-1$, stars to $\log (U)=-2$, open squares to $\log (U)=-2$, and open triangles to $\log (U)=-4$. It can be seen that Ly $\alpha$ and $2 \gamma$ luminosities scale with $P$ at low densities and Ly $\alpha$ is further enhanced at higher density. Case B values have been calculated using luminosity coefficients for $T_{\mathrm{e}}=30000 \mathrm{~K}$ and $n_{\mathrm{H}}=10^{2} \mathrm{~cm}^{-3}$ from Schaerer (2003). For simplicity we always compare the results to the low density case B limit.

The effect of "boosted" Ly $\alpha$ and $2 \gamma$ emission just discussed depends on the nebular metallicity. This is because more metals are present and more efficient cooling results in cooler gas than in the primordial metallicity case, decreasing the collisional effects for $\mathrm{H}$. The range over which these effects take place is discussed below.

\subsubsection{Density effects}

Figure 10 also shows that $\operatorname{Ly} \alpha$ is further enhanced at high densities at the expense of two-photon emission, the sum of the two luminosities being essentially constant if all other parameters are kept fixed. Collisional mixing of the relative populations of the $n=2$ levels between $2^{2} \mathrm{~S}$ and $2^{2} \mathrm{P}$ of hydrogen alter the relative importance of the rates of $\mathrm{Ly} \alpha$ and two-photon transitions resulting in increased line emission relative to the $2 \gamma$ continuum.
To correct for this effect under case B assumptions, Panagia (1973, see also Stiavelli 2009) writes $L(\operatorname{Ly} \alpha)=Q(\mathrm{H}) \times h v_{\text {Ly } \alpha} \times$ $f_{\text {coll }}$ (cf. Eq. (1)), where

$f_{\text {coll }} \approx \frac{1+a \times n_{\mathrm{p}}}{1.5+b \times n_{\mathrm{p}}}$

with $a=b=1.35 \times 10^{-4}$, and where $n_{\mathrm{p}}$ is the proton density. In this way the factor $f_{\text {coll }}$ ranges from $2 / 3 \approx \alpha_{2^{2} \mathrm{p}}^{\text {eff }} / \alpha_{\mathrm{B}}$ for low densities to $f_{\text {coll }}=1$ for high densities. Under these assumptions, the two-photon continuum luminosity is $L(2 \gamma)=Q(\mathrm{H}) \times h v_{\text {Ly } \alpha} \times$ $\left(1-f_{\text {coll }}\right)$.

To derive the corresponding numerical factor from our detailed photoionization models and to separate the density effect from the enhancement factor $P$ found above, we define $\tilde{f}_{\text {coll }}$ through:

$L(\mathrm{Ly} \alpha)=\left(1-f_{\text {esc }}\right) Q(\mathrm{H}) \times h v_{\text {Ly } \alpha} \times P \times \tilde{f}_{\text {coll }}$. 


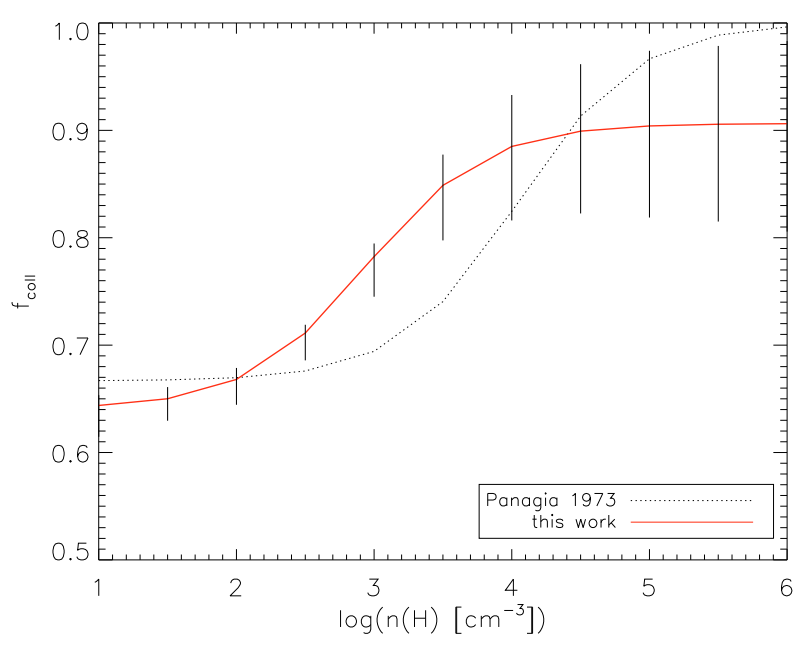

Fig. 11. $f_{\text {coll }}$ factor accounting for the density effects. The red line represents the 3-parameter fit obtained on the basis of the photoionization modeling $\left(\tilde{f}_{\text {coll }}\right)$ to be used in formula (8) to calculate $L(\operatorname{Ly} \alpha)$. At each density, vertical lines show the spread in $\tilde{f}_{\text {coll }}$ values arising from the different $T_{\mathrm{bb}}$ and $U$ models used: models with higher $T_{\mathrm{bb}}$ and lower $U$ have higher $\tilde{f}_{\text {coll }}$ values. The fit has been made for primordial nebular abundances and the black dotted line is $f_{\text {coll }}$ from Panagia (1973).

The corresponding values of $\tilde{f}_{\text {coll }}$, obtained for our grid of models for primordial nebulae are plotted in Fig. 11 as a function of the input hydrogen density. Also shown is, for comparison, Panagia's expression (Eq. (7)) assuming $n_{\mathrm{p}}=n_{\mathrm{H}}$. Notice that $\tilde{f}_{\text {coll }}$ presented here is not derived from the Ly $\alpha$ and $2 \gamma$ luminosities (which ranges from 2/3 to 1 as in Panagia's formula) but it is the fitting formula which has been calculated by comparing the right hand side of Eq. (8) with our CLOUDY results.

For convenience, we derive the following 3-parameter fit:

$\tilde{f}_{\mathrm{coll}} \approx \frac{1+a \times n_{\mathrm{H}}}{b+c \times n_{\mathrm{H}}}$,

with $a=1.62 \times 10^{-3}, b=1.56$ and $c=1.78 \times 10^{-3}$. The coefficients obtained here differ from those of Panagia (1973) due to the fact that we have used a suite of photoionization models covering a wide range of $T_{\mathrm{bb}}$ and $U$ resulting in different $T_{\mathrm{e}}$ and a different balance between processes in the nebula. However, the change in $\tilde{f}_{\text {coll }}$ has little effect on the values of $L(\operatorname{Ly} \alpha)$ for low density. We use $\tilde{f}_{\text {coll }}$ defined in this way for convenience since it allows us to reproduce the CLOUDY results. As Fig. 11 shows, our fit formula allows us to describe density effects with an accuracy of $\pm 10 \%$ at high density and somewhat better at low $n_{\mathrm{H}}$

The black dashed lines in Fig. 10 , showing $P \times \tilde{f}_{\text {coll }} / \tilde{f}_{\text {coll }}$ $\left(\log \left(n_{\mathrm{H}}=1\right)\right)$, show how well our analytical prediction for Ly $\alpha$, including also density effects, works at primordial metallicity.

\subsubsection{Applicable metallicity range}

The effect of collisional excitations, allowing for photoionization of $\mathrm{H}$ from excited states, depends on the electron temperature of the nebula, and hence on its metallicity. To find the range of nebular metallicity where our analytic expressions for $L(\operatorname{Ly} \alpha)$ holds, we have computed model grids at different metallicities. We can then ascertain how much our fit to the primordial case deviates from CLOUDY models as a function of metallicity.

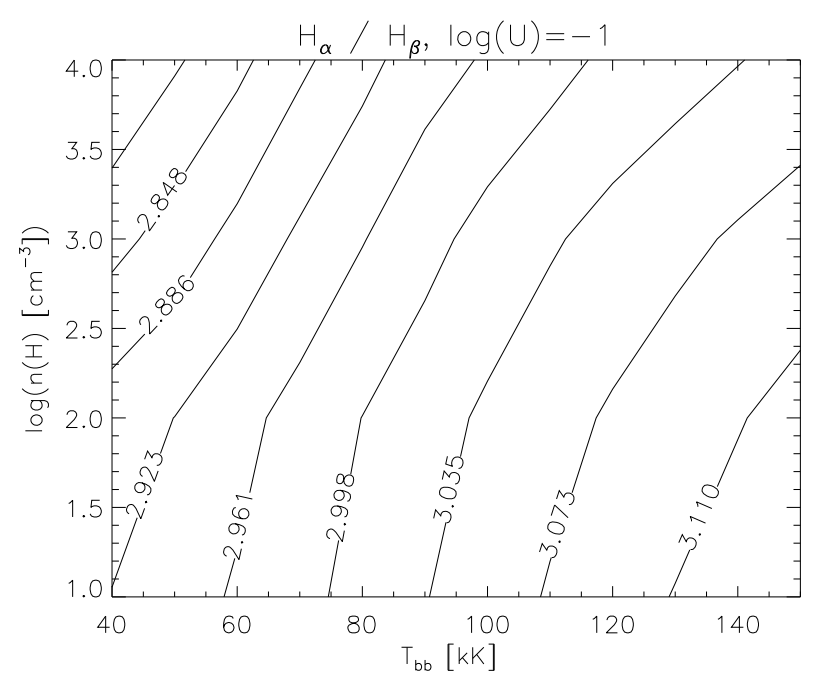

Fig. 12. The Balmer decrement as a function of $T_{\mathrm{bb}}$ and $n_{\mathrm{H}}$ for primordial metallicity case. Note that $\mathrm{H} \alpha$ is boosted for high $T_{\mathrm{bb}}$ due to collisional excitation of hydrogen.

The critical metallicity where the luminosity obtained by our fit deviates on average (for the entire grid of models) by $10 \%$ from the correct one is $Z_{\text {coll }} \approx 0.03 Z_{\odot}$. At higher metallicities, cooler nebulae are produced and hence collisional effects play a smaller role in boosting hydrogen emission. However, the effect only decreases gradually for higher metallicities and depends on the detailed condition of the gas. For instance, in our simulations for $Z_{\text {neb }}=0.05 Z_{\odot}$, the average deviation of the results obtained with our formula (primordial case) with respect to the CLOUDY results is $13 \%$, while for $Z_{\text {neb }}=0.1 Z_{\odot}$ it is $20 \%$. The value of the "transition" metallicity $Z_{\text {coll }} \approx 0.03 Z_{\odot}$ below which Ly $\alpha$ (and the $2 \gamma$ continuum) are significantly boosted (modified) by departures from case B, should therefore only be taken as an indicative value. Tailored photoionization models are necessary for more accurate predictions.

\subsection{Other $H$ lines}

The luminosities of other hydrogen lines are close to their case B predictions as is shown in Fig. 10 for $\mathrm{H} \alpha$ (green symbols) and $\mathrm{H} \beta$ (blue symbols). They are basically independent of $U$.

These lines are used to calculate the Balmer decrement which is conventionally used to measure the extinction. The Balmer decrement $(\mathrm{H} \alpha / \mathrm{H} \beta)$ obtained from our grid of models for $\log (U)=-1$ is shown in Fig. 12. It increases towards higher $T_{\mathrm{bb}}$ and exceeds the values commonly used, for example 2.86 for $n_{\mathrm{e}}=10^{2} \mathrm{~cm}^{-3}$ and $T_{\mathrm{e}}=10 \mathrm{kK}$ (Dopita \& Sutherland 1996). The reason for the enhanced $\mathrm{H} \alpha / \mathrm{H} \beta$ ratio is the collisional excitation of hydrogen, one of the contributors to enhanced Ly $\alpha$ and $2 \gamma$ emission. This effect (see Osterbrock 2006) occurs when photoelectrons carrying enough energy when colliding with $\mathrm{H}$ atoms, excite higher levels followed by radiative cascade. Enhanced $\mathrm{H} \alpha$ emission increases the Balmer decrement which must be accounted for when calculating the extinction. An enhanced Balmer decrement was already found, e.g. in tailored photoionization models for the metal-poor galaxy I $\mathrm{Zw}$ 18 (Davidson \& Kinman 1985; Stasińska \& Schaerer 1999) and other giant H II regions (cf. Luridiana et al. 2003), and has also been addressed by Luridiana (2009). 


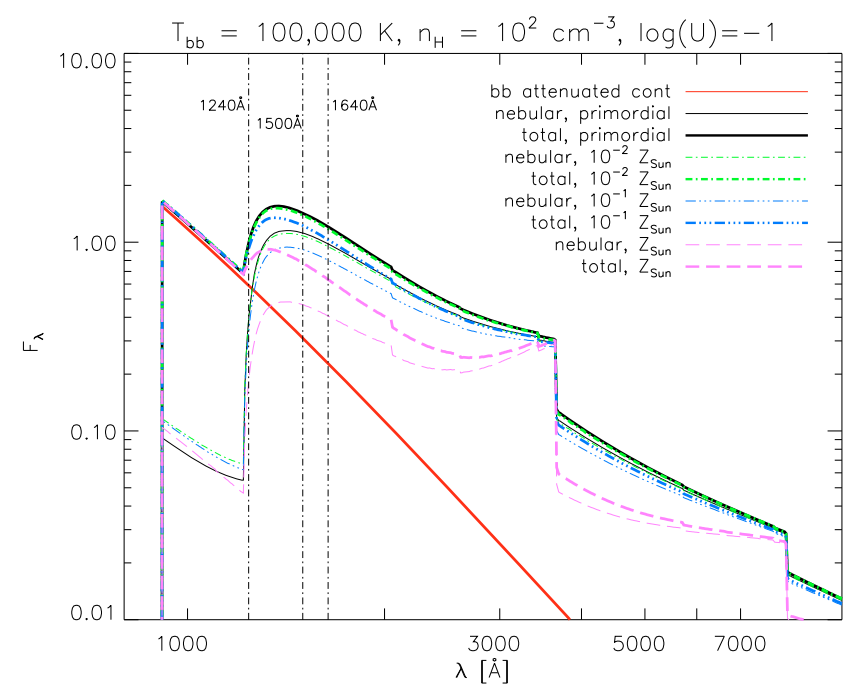

Fig. 13. Continuum of $T_{\mathrm{bb}}=100000 \mathrm{~K}$ models as a function of the nebular metallicity for a constant density and ionization parameter. The red solid line represents the transmitted stellar (black body) continuum. The thin lines are nebular only contributions to the continuum. The thick lines are the total continuum emission (stellar + nebular). The first model is normalized at $1240 \AA$ and the rest are rescaled accordingly.

\subsection{He II lines}

The He II $1640 \AA$ line is of a particular interest since it has been considered as one of the signatures of Pop III or very metal-poor stars, which are expected to have a very hard ionizing spectrum emitting copious $\mathrm{He}^{+}$ionizing photons. Figure 10 (orange symbols) shows He II $1640 \AA$ emission line luminosities relative to their case B values, calculated for the same $T_{\mathrm{e}}$ and $n_{\mathrm{e}}$ as the hydrogen lines. For lower ionization parameters, the line becomes weaker. This is due to an effect already discussed by Stasińska \& Tylenda (1986) in the context of planetary nebulae. Photons with sufficient energy to ionize $\mathrm{He}^{+}$are also able to ionize hydrogen. When calculating the luminosity of He II in synthesis models, it is assumed that every photon with an energy $>54 \mathrm{eV}$ ionizes one $\mathrm{He}^{+}$ion. The absorption of some high energy photons by $\mathrm{H}$ atoms results in a decrease in the number of photons available for producing He II emission. The effect becomes significant at low ionization parameters since then the $\mathrm{He}^{+} / \mathrm{H}^{0}$ fraction decreases and the probability of the high energy photon being absorbed by a $\mathrm{H}$ atom rather than by $\mathrm{He}^{+}$is higher. This means that the synthesis models give an upper limit for the He II luminosity. We have not found a simple analytical prescription to account for the effect of this process on the He II luminosity.

The behaviour of the equivalent width of He II is discussed in Sect. 5.

\subsection{Nebular continuum emission}

Nebular emission arising in $\mathrm{H}$ II regions can contribute significantly to the measured spectrum. The total nebular continuum is the sum of the free-free, free-bound and $2 \gamma$ continua of hydrogen and helium. Several nebular parameters affect its shape. It depends on the electron temperature, ionization parameter, nebular metallicity and particle density in the gas, as e.g. illustrated by Bottorff et al. (2006). In Fig. 14 we show the density dependence of the nebular continuum, which mainly affects the $2 \gamma$ emission and hence also the shape of the continuum between Ly $\alpha$ and the Balmer jump. Figure 13 shows the dependence on the nebular

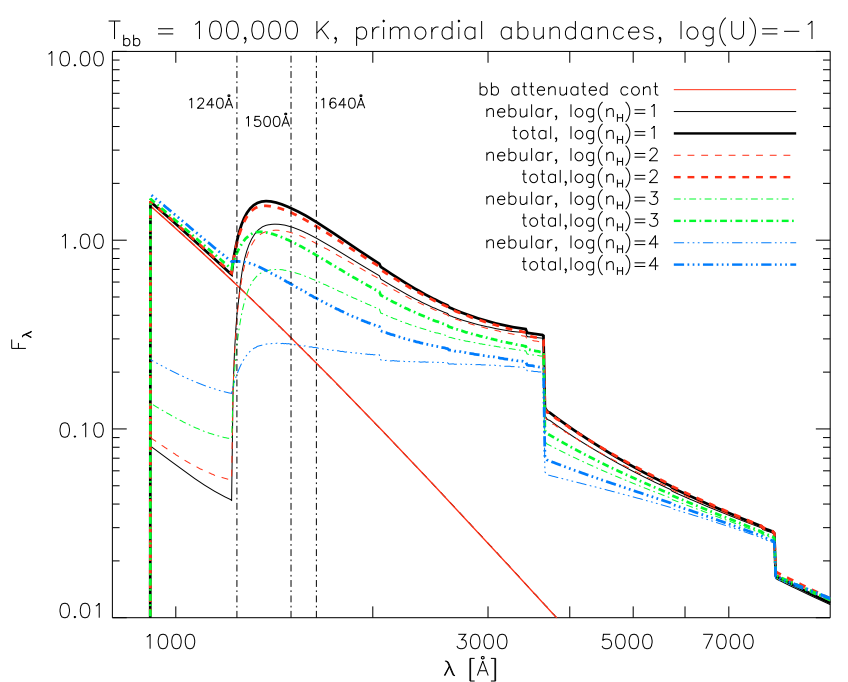

Fig. 14. Continuum of $T_{\mathrm{bb}}=100000 \mathrm{~K}$ models as a function of the hydrogen number density at the primordial metallicity and constant ionization parameter. The red solid line represents the transmitted stellar (black body) continuum. The thin lines are only nebular contributions to the continuum. The thick lines are the total continuum emission (stellar + nebular). At higher densities $2 \gamma$ continuum gets destroyed due to collisions. First model normalized at $1240 \AA$ and the rest rescaled accordingly.

metallicity. For higher metallicities, the increased cooling lowers the total emission in the nebular continuum, and its shape is altered as expected from atomic physics (see Bottorff et al. 2006) due to the decrease of the average electron temperature at higher metallicity. Evolutionary synthesis models such as ours, cannot properly describe the variety of shapes and strengths of the nebular continuum shown here, since they rely on simple assumptions such as constant nebular density and temperature. Again, tailored photoionization models are necessary for more accurate predictions.

\section{Photoionization models for realistic SEDs}

In this Section we show how to relate realistic SEDs obtained e.g. from evolutionary synthesis models with the results from the photoionization models discussed above, which used black body spectra. Finally, we show the updated predictions for the equivalent widths of Ly $\alpha$ and He II $\lambda 1640$ lines obtained from our CLOUDY models.

\subsection{How to connect realistic SEDs with black body calculations}

Since the number of $\mathrm{H}$ ionizing photons and their mean energy are the main parameters determining the luminosity of the $\mathrm{H} \mathrm{re-}$ combination lines and of the $2 \gamma$ continuum, $Q(\mathrm{H})$ and $P$ suffice to compute these quantities from arbitrary SEDs. To account also for density effects, Eqs. (8) and (9) can be used to yield the correct Ly $\alpha$ luminosity. Numerical tests using SEDs described in Sect. 3 show the validity of this result, typically within $2-3 \%$. For black bodies the relation between $P$ and $T_{\mathrm{bb}}$ is given by

$T_{\mathrm{bb}}[\mathrm{kK}]=-53.42+69.85 P$

to a good approximation. The black body temperature $T_{\mathrm{bb}}(P)$ corresponding to the more realistic SED is thus easily determined. 

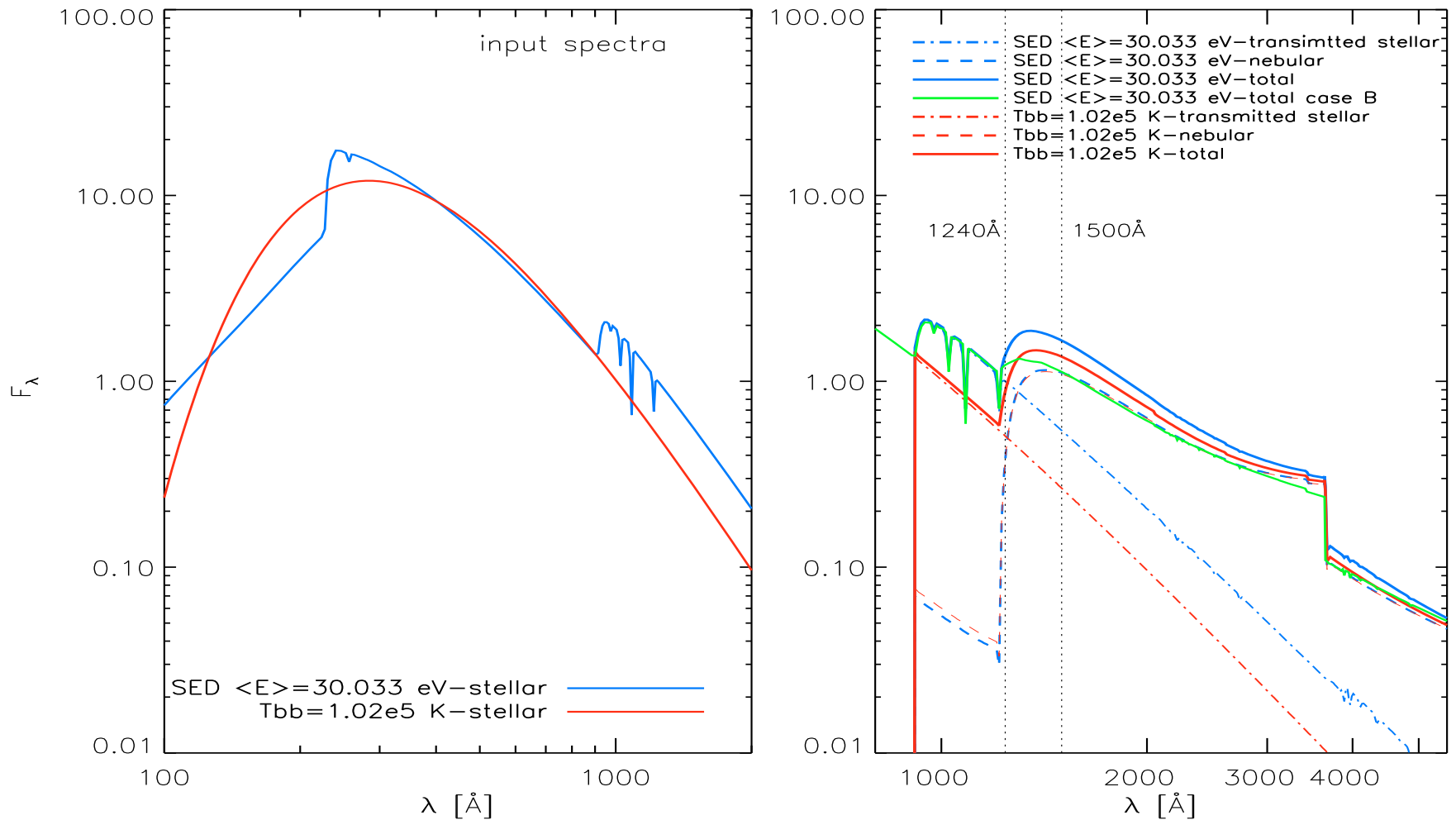

Fig. 15. Left: comparison of input stellar spectra for photoionization models showing a realistic SED (blue line; Pop III, Salpeter IMF $1-100 M_{\odot}$ at zero age) and the corresponding black body spectrum (red line) with the same average photon energy $(\bar{E}=30.033 \mathrm{eV})$ in the Lyman continuum. The SED model is normalized at $1240 \AA$ and the rest rescaled to match the same ionizing flux $Q(\mathrm{H})$. Right: input stellar spectra (dash-dotted, same colours as left panel) and predicted nebular and total continua from our CLOUDY models for $\log (n(\mathrm{H}))=1 \mathrm{~cm}^{-3}$ and $\log (U)=-1$ (solid lines, using the same colours as in left panel; cf. also inset for symbols). The green solid line shows the total (stellar + nebular) SED from our evolutionary synthesis model assuming constant nebular density and temperature and case B. Notice the difference between case B and the CLOUDY result due to enhanced $2 \gamma$ emission.

For other quantities, such as the He II line luminosity, the correspondence between black bodies and other SEDs is different. For example, since the relative line ratios of $\mathrm{He} \mathrm{II} / \mathrm{H}$ lines depend to first order on the relative number of $\mathrm{He}^{+} / \mathrm{H}$ ionizing photons, the black body with the same hardness $Q\left(\mathrm{He}^{+}\right) / Q(\mathrm{H})$ is the most appropriate. For black body spectra one has, to a good approximation:

$$
\begin{aligned}
T_{\mathrm{bb}}[\mathrm{kK}]= & 314.6+382.4 x+268.7 x^{2} \\
& +103.3 x^{3}+20.12 x^{4}+1.545 x^{5},
\end{aligned}
$$

where $x=\log \left(Q\left(\mathrm{He}^{+}\right) / Q(\mathrm{H})\right)$. Again, we have tested a number of models and confirmed that one can get the same luminosity (within a few\%) using SEDs and corresponding black body models, for otherwise identical nebular parameters. Differences of $\sim 10 \%$ can appear in case of significantly (several eV) different values of $P$. In principle not only the stellar $Q\left(\mathrm{He}^{+}\right) / Q(\mathrm{H})$ ratio determines the $\mathrm{He}$ II emission, but also the conditions in the gas. Since $P$ establishes the electron temperature, significant differences in $P$ result in different luminosity coefficients (recombination rates and emissivities).

In Fig. 15 we show such a comparison between a realistic SED from our synthesis models (Pop III, Salpeter IMF $1-100 M_{\odot}$ at zero age, instantaneous burst) and the appropriate black body, chosen such as to reproduce the correct $\mathrm{H}$ line luminosities and nebular continuum. Here the average photon energy in the Lyman continuum is $\bar{E}=30.033 \mathrm{eV}$, hence $T_{\mathrm{bb}}=102 \mathrm{kK}$. The input spectra, shown on the left panel, are scaled to the same total Lyman continuum flux $Q(\mathrm{H})$. The nebular parameters are $\log \left(n_{\mathrm{H}}\right)=1 \mathrm{~cm}^{-3}, \log (U)=-1$, and primordial composition.

Several interesting points are illustrated with this figure. First, as the left panel shows, it must be remembered that realistic SEDs show a Lyman break due to the hydrogen opacity in the atmosphere of the hot stars responsible for the flux. For young ages this break corresponds typically to a flux increase by $\sim 0.2-0.3$ dex at $912 \AA$, as discussed e.g. by Schaerer $(2003)^{4}$. In consequence, calculations relying on black body spectra underestimate the observable UV continuum, as shown in the right panel of Fig. 15, leading to non-negligible differences e.g. for equivalent widths predictions of emission lines (cf. below). In Fig. 15 (right panel) we also show the stellar + nebular SED predicted by our synthesis models assuming constant nebular

4 The amplitudes of the Lyman break, $912^{+} / 912^{-}$, given in Schaerer (2003) for very low metallicities are correct, but misleading. Indeed, with the definition adopted there, the $912^{+}$flux average (over 1080-1200 ̊) includes strong absorption from the He II $\lambda 1084$ line. This leads to values of $912^{+} / 912^{-}<1$ despite the fact that all models show a Lyman break in absorption. 

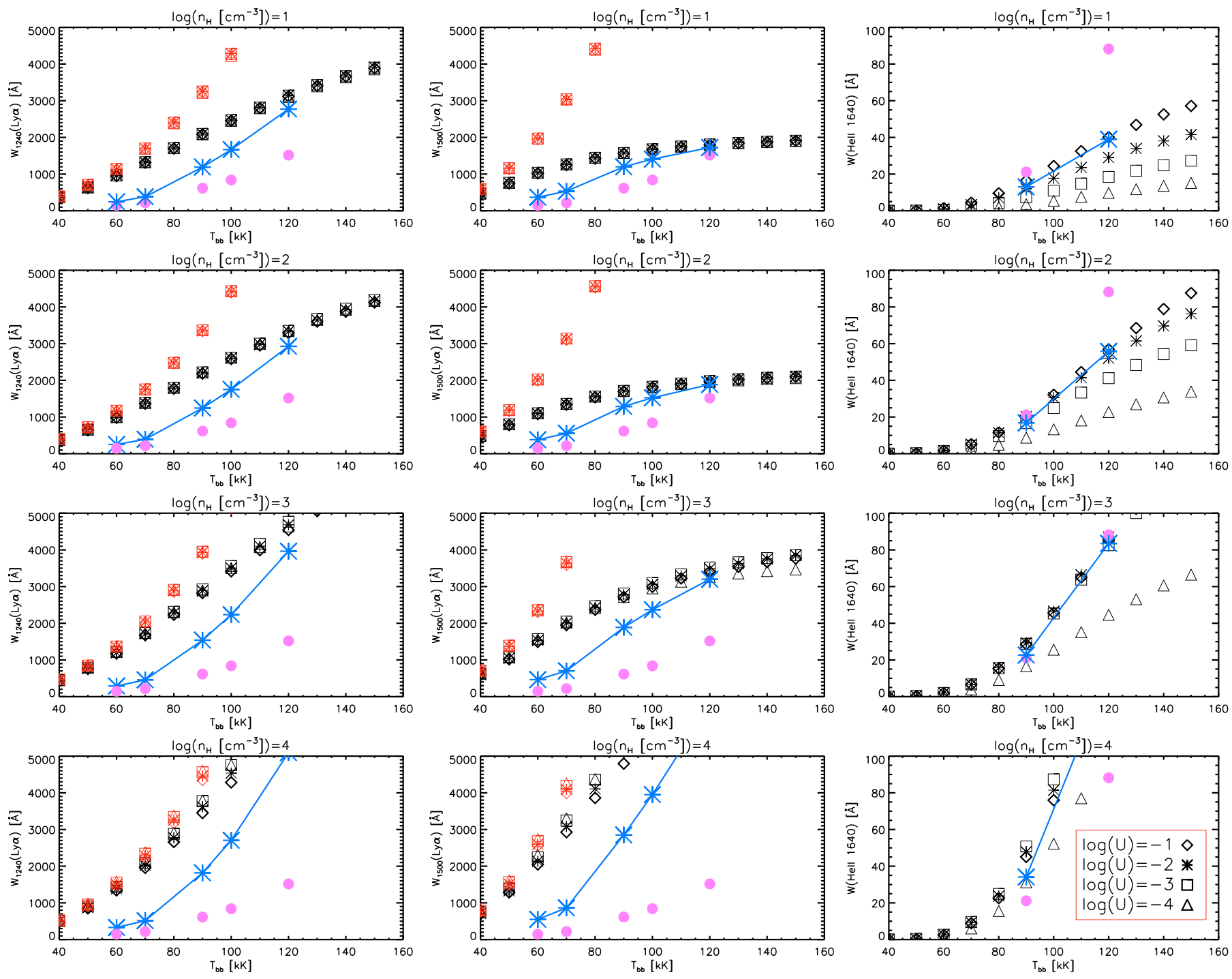

Fig. 16. Equivalent widths ( $\left.W_{\lambda}\right)$ of Ly $\alpha$ at 1240 (Col. 1) and $1500 \AA$ (Col. 2) and He II $\lambda 1640$ (Col. 3) for a primordial metallicity nebula at different densities and ionization parameters (marked with different symbols as defined in the inset). The red symbols show an upper limit for $W_{\lambda}(\mathrm{Ly} \alpha)$ since they are calculated using only stellar (black body) continuum. Blue symbols - the most realistic predictions - show the results obtained for the corresponding stellar population SEDs (models with the same $P$ in the case of $\mathrm{Ly} \alpha$ and with the $\operatorname{same} \log \left(Q\left(\mathrm{He}^{+}\right) / Q(\mathrm{H})\right)$ for the He II analysis, for simplicity only models with $\log (U)=-1$ are shown) and the pink ones their case B predictions. Notice the dependence of the He II $\lambda 1640$ equivalent width on the ionization parameter and the discrepancy between its case B and photoionization model predictions for real SEDs.

density and temperature and case B (green line). The differences between this and the full CLOUDY model are mainly due to the boosting of the $2 \gamma$ continuum discussed earlier, and to varying $n_{\mathrm{e}}$ and $T_{\mathrm{e}}$.

\subsection{Ly $\alpha$ equivalent width predictions}

The measurement of the equivalent width of $\operatorname{Ly} \alpha$ is both observationally and interpretationally difficult, since the continuum around it is affected on one side at high redshifts by Ly $\alpha$ forest absorption and on the other by an unknown combination of $2 \gamma$ continuum and starlight. Furthermore, when not measured from spectroscopy, it is common practice to measure $W(\operatorname{Ly} \alpha)$ via a line to continuum ratio with the continuum estimated at another (usually longer) wavelength $\lambda_{\text {cont }}$, e.g. $W_{\lambda}=L_{\text {line }} / F_{\lambda_{\text {cont }}}$. Different methods to measure $W(\operatorname{Ly} \alpha)$ have been reviewed by Hayes \& Östlin (2006). To illustrate the possible impact or uncertainty related to the way the continuum is estimated we subsequently plot Ly $\alpha$ equivalent widths predicted from the CLOUDY models using the continuum at both 1240 and $1500 \AA$ as a reference.

Figure 16 shows the predictions for $W(\operatorname{Ly} \alpha)$ from all CLOUDY models for primordial composition, as a function of $T_{\mathrm{bb}}$, ionization parameter and hydrogen density. Black symbols show the predictions using black body spectra and accounting for the nebular continuum; red symbols the same but neglecting the nebular continuum. Blue symbols show the results from CLOUDY models using SEDs from our Pop III synthesis models for zero age populations, plotted at the corresponding $T_{\mathrm{bb}}(P)$ value (see above). Pink symbols show $W(\mathrm{Ly} \alpha)$ predicted from our standard synthesis models neglecting the boost of Ly $\alpha$ (case B departure).

Overall $W(\operatorname{Ly} \alpha)$ increases with the $T_{\mathrm{bb}}$ or equivalent since the $\operatorname{Ly} \alpha$ luminosity $(\propto Q(\mathrm{H}) \times P)$ increases more rapidly than the continuum flux close to $\operatorname{Ly} \alpha$. For very hot models and if $1500 \AA$ is taken as a reference for the continuum, $W(\operatorname{Ly} \alpha)$ tends 


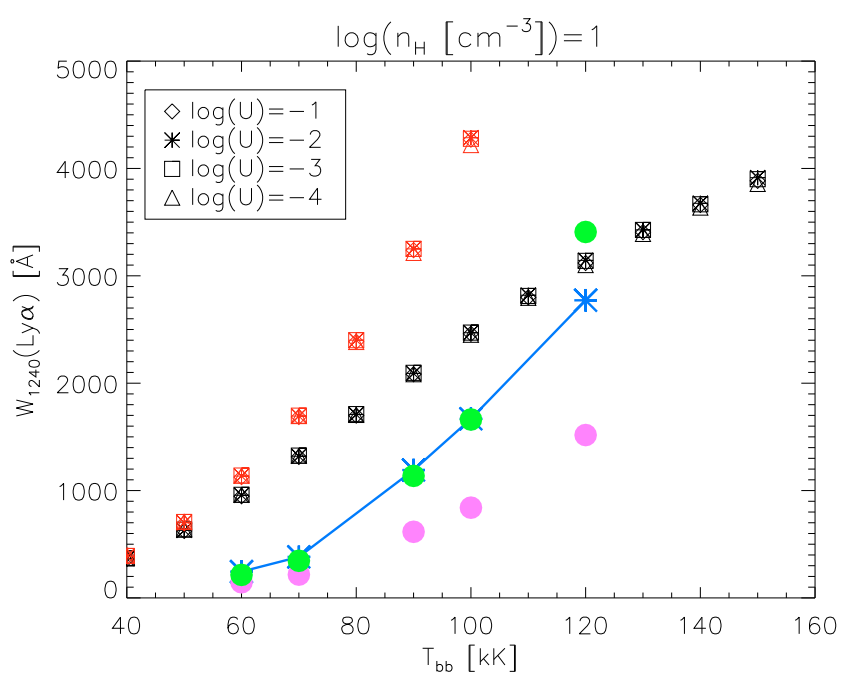

Fig. 17. Equivalent widths $\left(W_{\lambda}\right)$ of Ly $\alpha$ at $1240 \AA$ (first column, second row of Fig. 16). All the symbols as in Fig. 16. Additionally, the green symbols show the case B SED results (pink symbols) corrected using our formula (first order correction; only the enhancement in the line flux is taken into account, there is no correction for the continuum). The difference coming from not correcting for the nebular continuum enhancement is visible for the hottest model.

to a maximum value (here $\sim 2000 \AA$, as shown in Col. 2) since line emission and the dominating nebular continuum scale in the same manner. For the reasons discussed above (absence of the Lyman break), predictions based on black body spectra (black and red symbols) overestimate $W(\operatorname{Ly} \alpha)$ compared to models including more realistic stellar SEDs (in blue). Differences between black body models including or neglecting the nebular continuum (black and red) decrease with increasing ISM density, because of the decrease of the $2 \gamma$ continuum. This also explains the decreasing difference between $W(\operatorname{Ly} \alpha)$ using 1240 and $1500 \AA$ as a reference, when $n_{\mathrm{H}}$ increases. Last, but not least, $W(\operatorname{Ly} \alpha)$ predicted by our standard synthesis models for the ZAMS (pink symbols) fall below the more accurate CLOUDY predictions (blue), since the latter account for case B departures. Once applying the simple correction given by Eq. (4) one obtains the result which takes into account the increase in Ly $\alpha$ line flux. However, that is only the first order correction which does not include the difference coming from the continuum, mainly a similar increase in the $2 \gamma$ continuum. In cases when the continuum is measured around Ly $\alpha(1240 \AA)$ stellar flux is dominant so the correction described above gives a good estimate of the correct $W(\operatorname{Ly} \alpha)$ value (see Fig. 17).

\subsection{He II $\lambda 1640$ equivalent width}

The right column in Fig. 16 shows the predicted He II $\lambda 1640$ equivalent widths for the same CLOUDY models except that $T_{\mathrm{bb}}(x)$ from Eq. (11) is used here. The nebular continuum predicted at $1640 \AA$ is included in all calculations of $W(1640)$. In contrast to $\operatorname{Ly} \alpha$, the He II equivalent width depends on the ionization parameter, since the line luminosity changes due to the "Stasinska-Tylenda effect". For high ISM densities $W(1640)$ increases due to the decrease of the nebular continuum. Differences between models assuming black body spectra (black symbols) and realistic SEDs (blue) are minor for $W(1640)$ as long the nebular continuum dominates at these wavelengths. This is true for sufficiently hot spectra $(\gtrsim 80 \mathrm{kK})$. Compared to predictions from our standard evolutionary synthesis models (pink symbols) the He II $\lambda 1640$ equivalent widths predicted by CLOUDY are significantly lower for $T_{\mathrm{bb}} \gtrsim 100 \mathrm{kK}$. This is due to two effects, the Stasinska-Tylenda effect reducing the line flux, and the increase of the $2 \gamma$ nebular continuum. At lower $T_{\mathrm{bb}}$, case B predictions give an upper limit (corresponding to our highest ionization parameter case) for He II $\lambda 1640$. Only for a high ionization parameter and/or high density do photoionization models predict $W(1640)$ values as high as those given by Schaerer (2003).

\subsection{Summary: correction of evolutionary synthesis model results for departures from case $B$}

In this section we give a recommended step-by-step procedure which allows the correction of the synthesis models for the departures from case B in ionization bounded regions (i.e. with no leakage of Lyman continuum photons), relevant especially for the case of very hot stars and very low metallicities. For L(Lya):

- take the SED (function of age, IMF, ...);

- get (or compute) the mean energy of Lyman continuum photons, $P$, in units of $13.6 \mathrm{eV}$;

- compute the "collisional" factor $\tilde{f}_{\text {coll }}$ from our fit formula (Eq. (9));

- multiply the synthesis model result $L(\operatorname{Ly} \alpha)$ by $P * \tilde{f}_{\text {coll }} /(2 / 3)$ (Eq. (4)).

For $\mathrm{W}(\operatorname{Ly} \alpha)$ : divide the equivalent width obtained by the synthesis model of interest by $2 / 3$ and multiply it by $P * \tilde{f}_{\text {coll }}$. This provides the first order correction for $W(\operatorname{Ly} \alpha)$, neglecting the increase of the nearby nebular continuum. An example following this procedure is shown by the green symbols in Fig. 17.

For He II recombination lines: no simple correction is possible. Synthesis models give an upper limit for the luminosity, an upper limit for equivalent width up to $\log \left(Q\left(\mathrm{He}^{+}\right) / Q(\mathrm{H})\right) \sim-1.2$ for the primordial metallicity case and overestimate it for the highest $Q\left(\mathrm{He}^{+}\right) / Q(\mathrm{H})$ cases (low density) due to the lack of accounting for enhanced $2 \gamma$ emission.

\section{Discussion and implications}

\subsection{Dependence on model assumptions}

All our CLOUDY models have been computed for ionization bounded nebulae corresponding to no escape of Lyman continuum photons, i.e. $f_{\text {esc }}=0$. For density (or matter) bounded cases with $f_{\text {esc }}>0$, quantities such as hydrogen recombination line luminosities and the luminosity of the nebular continuum longward of $\operatorname{Ly} \alpha$ can be rescaled to first order by scaling with $\left(1-f_{\text {esc }}\right)$, as already introduced in Eq. (8). Such a scaling is appropriate since the corresponding emissivities are approximately constant across the nebula. However, since $\mathrm{He}^{+}$ recombination lines (if present) are emitted only in the innermost parts of the nebula, He II line luminosities will not be reduced in density bounded regions, as long the radius of the $\mathrm{He}^{+}$ sphere remains smaller than that of density bounded H II region. These behaviours are also expected in non-spherical regions, as demonstrated e.g. by Johnson et al. (2009). In consequence, both $L(\operatorname{Ly} \alpha)$ and $W(\operatorname{Ly} \alpha)$ will decrease with increasing Lyman continuum escape (although the latter not proportionally with $f_{\text {esc }}$ ), whereas the opposite will be true for $W(\mathrm{He}$ II 21640$)$ and for other He II lines, where the underlying continuum is mostly of nebular origin. In other words, the decrease of $W(\mathrm{He}$ II 21640$)$ 
found in this paper due to competition with $\mathrm{H}$ ionizing photons, may be mitigated in objects with significant leakage of Lyman continuum radiation, leading again to higher $\mathrm{He}$ II equivalent widths (cf. Schaerer 2002, 2003). For other effects of "leaking", i.e. star-forming galaxies with density bounded $\mathrm{H}$ II regions, see the photoionization models of Inoue (2010).

We shall now discuss some implications of our modeling for the interpretation of the hydrogen and helium emission lines and the nebular continuum observed in high redshift emission line galaxies.

\subsection{Lyman continuum output}

As metallicity decreases, the Lyman continuum output of the stellar population, both relative to the observed stellar UV continuum longward of Ly $\alpha$ and per unit stellar mass, increases. The amount of this increase depends obviously also on the detailed shape of the IMF. For example, for a Salpeter IMF with a uniform upper mass cut-off of $100 M_{\odot}$, the ionizing photon rate per unit UV luminosity, $Q_{\mathrm{H}} / L_{1500}$, increases by a factor 3 approximately between solar an zero metallicity. For the most extreme IMF considered here this increase is up to a factor 10 (see Fig. 1). The effect of this is to increase the line and nebular continuum emission relative to any observable stellar continuum. In extreme cases (e.g., Raiter et al. 2010) the nebular emission may completely dominate the UV/visible/NIR spectrum. Also, if low metallicity objects and/or different IMFs are relevant for the sources of cosmic reionization, their intrinsic Lyman continuum flux, generally estimated from UV restframe observations, may need to be revised accordingly.

\section{3. $S F R(U V)$}

The estimation of SFR from the level of the UV continuum (cf. Kennicutt 1998) is based on evolutionary synthesis models assuming "standard" stellar populations and IMFs. At metallicities below solar, the output of observable UV continuum radiation (i.e. typically at $1500 \AA$ restframe) per unit stellar mass increases somewhat, due to the increasing average temperature of the stars (cf. Fig. 4). A maximum in $L_{1500}$ is typically reached at $Z / Z_{\odot} \sim 1 / 100$. Below this, the average spectrum shifts more strongly into the Lyman continuum, leading to a decrease of stellar radiation in the observable UV, which is, however, compensated by increasing nebular emission. The net result, illustrated in Fig. 4 for constant SFR, is that star-formation rates derived from the UV may need to be revised downward at low metallicities with respect to the "standard" calibration of Kennicutt (1998), but typically by less than a factor 2 .

\section{4. $L y \alpha$}

One of the main findings of our study is the enhancement of Ly $\alpha$ emission at low metallicities with respect to the commonly used case B value. This departure from case B occurs even at moderaly low metallicity, say $Z / Z_{\odot} \lesssim 1 / 10$, and becomes more important for lower $Z$. In consequence, higher intrinsic Ly $\alpha$ line luminosities relative to the UV continuum (to $L_{\mathrm{bol}}$, SFR, or to another measure of the rate of massive star-formation) are expected and higher Ly $\alpha$ equivalent widths. Even higher values of $W(\operatorname{Ly} \alpha)$ can be obtained in regions with a high ISM density, where Ly $\alpha$ emission can further be "boosted" at the expense of the 2-photon nebular continuum (see Fig. 10).

With respect to our earlier predictions (cf. Schaerer 2003), $W(\operatorname{Ly} \alpha)$ is increased by a factor $2-2.5$ for Pop III objects, and by more than $70 \%$ at moderately low metallicity $\left(Z / Z_{\odot} \lesssim 1 / 20\right.$, cf. Fig. 5), for a given IMF. This increase of Ly $\alpha$ may help to understand objects with large $\operatorname{Ly} \alpha$ equivalent widths found in some surveys, without the need for recourse to unusual IMFs or to a clumpy ISM (cf. Malhotra \& Rhoads 2002; Dawson et al. 2004; Finkelstein et al. 2008).

Finally, the enhanced Ly $\alpha$ emission found here should represent good news for searches for very distant/early galaxies, since the intrinsic Ly $\alpha$ emission of metal-poor objects is shown to be considerably higher than previously thought, reaching up to $20-40 \%$ of $L_{\text {bol }}$ in Pop III dominated objects. Of course, the intrinsic $\operatorname{Ly} \alpha$ emission (and hence also $W(\operatorname{Ly} \alpha)$ ) will be lower in objects, having a non-zero escape of flux from the Lyman continuum. Furthermore, several processes exist (e.g. dust absorption, scattering out of the line-of-sight in the ISM and/or IGM), which will reduce the Ly $\alpha$ emission on the way from the source to the observer.

\subsection{He II emission}

The reduced He II emission found in this paper, compared to our earlier predictions assuming simple case B recombination, may indicate that ongoing searches for signatures of Pop III stars using this feature (see e.g. review by Schaerer 2008) could be more difficult than anticipated so far. The corresponding upper limits of Pop III star-formation rate density derived e.g. from the survey of Nagao et al. (2008) need then to be revised upwards.

The detection of the He II $\lambda 1640$ line indicates a significant number of high energy photons in the ionizing spectrum. This line has therefore been sought, but not detected so far at high- $z$ (Nagao 2008). Prescott et al. (2009) have detected this line from a $z=1.67$ spatially extended nebula (or Ly $\alpha$ blob) but have not been able to determine the nature of the ionizing source. We find that the line (and therefore the equivalent width) could be fainter than expected previously, due to its dependence on the ionization parameter. This suggests that deeper observations will be needed to detect it. Additionally, another difficulty comes from the fact that for the hottest (the highest $\left.Q\left(\mathrm{He}^{+}\right) / Q(\mathrm{H})\right)$ ratio) stars the strongest enhancement in $2 \gamma$ continuum is predicted which further decreases the measured $W(1640)$. However, if Pop III stars are present in objects with significant escape fractions in the Lyman continuum, the He II $\lambda 1640$ equivalent width may again be stronger, due to the reduced nebular continuum (cf. Sect. 6.1).

\subsection{Nebular continuous emission}

The overall nebular continuum emission is dependent on several nebular parameters and can result in a variety of spectral shapes (Figs. 13 and 14). Note that our simulations have been carried out in the absence of dust which would add another parameter affecting the shape of the continuum.

Proper photoionization modeling shows the importance of the $2 \gamma$ continuum produced in a nebula ionized by very hot stars. At low ISM density and primordial/low metallicity, it is boosted in the same way as Ly $\alpha$ and can completely dominate the nebular emission at 1216-1600 $\AA$. This can affect the broadband flux measurements and reduce the equivalent widths of UV emission lines, particularly He II. The strength of the nebular continuum and the expected variations of its spectral shape also indicate that measurements of the $\operatorname{Ly} \alpha$ equivalent width from photometry may be more uncertain than naively expected (cf. Hayes \& Östlin 2006).

As can be seen from Fig. 15, the slope of the UV continuum between 1300-2000 A obtained from our CLOUDY model is 
very similar to that predicted by the evolutionary synthesis models that use the case B assumption with constant electron density and temperature. When the enhanced $2 \gamma$ continuum is included, one cannot obtain significantly steeper slopes than found for this Pop III simulation.

\section{Conclusions}

Building on the earlier calculations of Schaerer (2002, 2003), we have computed evolutionary synthesis models for a wide range of metallicities from Pop III (zero metallicity) to solar metallicity covering a wider range of IMFs - including power laws and log-normal IMFs with different characteristic masses - than before. Using these synthesis models, we present the expected UV properties of star-forming galaxies, including their Lyman continuum fluxes, UV luminosity, and properties of the continuum (e.g. $\beta$-slopes), as well as properties of important emission lines such as Ly $\alpha$ and He II $\lambda 1640$ (see Sect. 3).

To investigate possible departures from the simple case B recombination theory assumed in many synthesis models, including ours, we have computed large grids of CLOUDY photoionization models for zero and low metallicity nebulae, using both black-body spectra and the SEDs predicted by our synthesis models (Sects. 4 and 5).

Our main conclusions from the photoionization models are the following:

- Due to departures from case B (collisional excitation and ionization from excited levels), both Ly $\alpha$ and $2 \gamma$ continuum emission can be significantly enhanced at low nebular densities. Their strengths are found to scale with the mean photon energy of the ionization source in the Lyman continuum.

- The equivalent width of Ly $\alpha$ can be larger than expected from case B calculations due to the line flux enhancement. The measurement can also be affected by $2 \gamma$ emission if the continuum is measured at longer wavelength.

- Further enhancement of $\operatorname{Ly} \alpha$ at the expense of $2 \gamma$ emission can occur due to collisional mixing between the hydrogen $2^{2} \mathrm{~S}-2^{2} \mathrm{P}$ levels at higher densities.

- He II emission line fluxes (and consequently their equivalent widths) can be significantly decreased due to their dependence on the ionization parameter. This could make searches for the He II $\lambda 1640$ line at high- $z$ more difficult.

- The enhancement of the $2 \gamma$ continuum and its dependence on nebular parameters can result in reduced equivalent widths of the UV emission lines and also change the UV restframe colours of high- $z$ galaxies.

Our results are of relevance to searches for, and interpretation of observations of, metal-poor and/or high- $z$ galaxies which may host the first stars that appeared in the Universe.

Acknowledgements. We thank Suzy Collin, Mike Fall, and Grazyna Stasińska for helpful and stimulating discussions. Some explorations of photoionization models were already undertaken earlier with Francois Ricquebourg and Michael Zamo. D.S. wishes to thank them here for their contributions. The work of D.S. is supported by the Swiss National Science Foundation. D.S. is thankful for support from the ESO visitor program, during which part of this work was done.

\section{References}

Balestra, I., Mainieri, V., Popesso, P., et al. 2010, A\&A, 512, A12

Bottorff, M. C., Ferland, G. J., \& Straley, J. P. 2006, PASP, 118, 1176

Bouwens, R. J., Illingworth, G. D., Oesch, P. A., et al. 2010, ApJ, 708, L69

Bromm, V., Kudritzki, R. P., \& Loeb, A. 2001, ApJ, 552, 464

Ciardi, B., Ferrara, A., Marri, S., \& Raimondo, G. 2001, MNRAS, 324, 381

Davidson, K., \& Kinman, T. D. 1985, ApJS, 58, 321

Dawson, S., Rhoads, J. E., Malhotra, S., et al. 2004, ApJ, 617, 707

Ferland, G. J., Korista, K. T., Verner, D. A., et al. 1998, PASP, 110, 761

Finkelstein, S. L., Rhoads, J. E., Malhotra, S., Grogin, N., \& Wang, J. 2008, ApJ, 678,655

Finkelstein, S. L., Papovich, C., Giavalisco, M., et al. 2010, ApJ, 719, 1250

Gnedin, N. Y., Kravtsov, A. V., \& Chen, H. 2008, ApJ, 672, 765

Hayes, M., \& Östlin, G. 2006, A\&A, 460, 681

Inoue, A. K. 2010, MNRAS, 401, 1325

Johnson, J. L., Greif, T. H., Bromm, V., Klessen, R. S., \& Ippolito, J. 2009, MNRAS, 399, 37

Kennicutt, Jr., R. C. 1998, ARA\&A, 36, 189

Larson, R. B. 1998, MNRAS, 301, 569

Luridiana, V. 2009, Ap\&SS, 324, 361

Luridiana, V., Peimbert, A., Peimbert, M., \& Cerviño, M. 2003, ApJ, 592, 846

Malhotra, S., \& Rhoads, J. E. 2002, ApJ, 565, L71

Nagao, T., Sasaki, S. S., Maiolino, R., et al. 2008, ApJ, 680, 100

Osterbrock, D. E., \& Ferland, G. J. 2006, Astrophysics of gaseous nebulae and active galactic nuclei

Panagia, N. 1973, AJ, 78, 929

Panagia, N. 2002, [arXiv:astro-ph/0209346]

Panagia, N. 2005, in The Initial Mass Function 50 Years Later, ed. E. Corbelli, F. Palla, \& H. Zinnecker, Astrophys. Space Sci. Library, 327, 479

Popesso, P., Dickinson, M., Nonino, M., et al. 2009, A\&A, 494, 443

Raiter, A., Fosbury, R. A. E., \& Teimoorinia, H. 2010, A\&A, 510, A109

Razoumov, A. O., \& Sommer-Larsen, J. 2010, ApJ, 710, 1239

Scalo, J. M. 1986, Fund. Cosmic Phys., 11, 1

Schaerer, D. 2002, A\&A, 382, 28

Schaerer, D. 2003, A\&A, 397, 527

Schaerer, D. 2008, in IAU Symp., ed. L. K. Hunt, S. Madden, \& R. Schneider, 255,66

Schaerer, D., \& Vacca, W. D. 1998, ApJ, 497, 618

Schaerer, D., \& Pelló, R. 2005, MNRAS, 362, 1054

Schaerer, D., \& Verhamme, A. 2008, A\&A, 480, 369

Schaerer, D., \& de Barros, S. 2010, A\&A, 515, A73

Schneider, R., Ferrara, A., Natarajan, P., \& Omukai, K. 2002, ApJ, 571, 30

Schneider, R., Ferrara, A., Salvaterra, R., Omukai, K., \& Bromm, V. 2003, Nature, 422, 869

Stasińska, G., \& Schaerer, D. 1999, A\&A, 351, 72

Stasińska, G., \& Tylenda, R. 1986, A\&A, 155, 137

Stiavelli, M. 2009, From First Light to Reionization: The End of the Dark Ages, ed. M., Stiavelli (Wiley)

Tumlinson, J. 2006, ApJ, 641, 1

Tumlinson, J., \& Shull, J. M. 2000, ApJ, 528, L65

Tumlinson, J., Giroux, M. L., \& Shull, J. M. 2001, ApJ, 550, L1

Vanzella, E., Cristiani, S., Dickinson, M., et al. 2005, A\&A, 434, 53

Vanzella, E., Cristiani, S., Dickinson, M., et al. 2006, A\&A, 454, 423

Vanzella, E., Cristiani, S., Dickinson, M., et al. 2008, A\&A, 478, 83

Vanzella, E., Giavalisco, M., Dickinson, M., et al. 2009, ApJ, 695, 1163

Wise, J. H., \& Cen, R. 2009, ApJ, 693, 984

Yamada, S. F., Sasaki, S. S., Sumiya, R., et al. 2005, PASJ, 57, 881 